\title{
Stability of Euclidean space under Ricci flow
}

\author{
Oliver C. Schnürer, Felix Schulze And Miles Simon
}

\begin{abstract}
We study the Ricci flow for initial metrics which are $C^{0}$ small perturbations of the Euclidean metric on $\mathbb{R}^{n}$. In the case that this metric is asymptotically Euclidean, we show that a Ricci harmonic map heat flow exists for all times, and converges uniformly to the Euclidean metric as time approaches infinity. In proving this stability result, we introduce a monotone integral quantity which measures the deviation of the evolving metric from the Euclidean metric. We also investigate the convergence of the diffeomorphisms relating Ricci harmonic map heat flow to Ricci flow.
\end{abstract}

\section{Introduction}

In this paper we investigate the evolution of a family of complete noncompact Riemannian manifolds $\left(\mathbb{R}^{n}, g(t)\right)$ under Ricci flow $(\mathrm{RF})$

$$
\begin{cases}\frac{\partial}{\partial t} g(t)=-2 \operatorname{Ric}(t), & \text { in } \mathbb{R}^{n} \times(0, \infty), \\ g(t) \rightarrow g_{0}, & \text { in } C_{\mathrm{loc}}^{0} \text { as } t \searrow 0,\end{cases}
$$

where $g_{0}$ is a given initial metric on $\mathbb{R}^{n}$. We study the long-term behavior as $t \rightarrow \infty$ of solutions to (RF) for initial metrics $g_{0}$ which are $C^{0}$-close to the standard Euclidean metric $h$, in standard coordinates $h_{i j}=\delta_{i j}$. For analytic reasons, it is convenient to study the Ricci harmonic map heat flow which is a variant of the Ricci-DeTurck flow

$$
\begin{cases}\frac{\partial}{\partial t} g_{i j}(x, t)=-2 R_{i j}(x, t)+\nabla_{i} V_{j}+\nabla_{j} V_{i} & \text { in } \mathbb{R}^{n} \times(0, \infty), \\ g_{i j}(t) \rightarrow\left(g_{0}\right)_{i j} & \text { in } C_{\text {loc }}^{0}\left(\mathbb{R}^{n}\right) \text { as } t \searrow 0\end{cases}
$$

where $V_{i}=g_{i k}\left({ }^{g} \Gamma_{r s}^{k}-{ }^{h} \Gamma_{r s}^{k}\right) g^{r s}$, for a flat metric $h, V_{i}=g_{i k}{ }^{g} \Gamma_{r s}^{k} g^{r s}$. If a family of metrics $(g(t))_{t \in(0, \infty)}$ solves (1.1), we call it a solution to the $h$-flow with initial metric $g_{0}$. Precise definitions are to be found in Section 2. Note that the $h$-flow (1.1) and the (RF) equation are equivalent up to diffeomorphisms [12] in the case that the initial metric is smooth, and $M$ is compact. 
Definition 1.1. Let $g_{1}$ and $g_{2}$ denote two Riemannian metrics on a given manifold. We say that $g_{1}$ is $\delta$-fair to $g_{2}$, if

$$
\delta^{-1} g_{2} \leq g_{1} \leq \delta g_{2}
$$

We call $g_{1} \varepsilon$-close to $g_{2}$, if $g_{1}$ is $(1+\varepsilon)$-fair to $g_{2}$.

We denote with $\mathcal{M}^{k}\left(\mathbb{R}^{n}, I\right)$ the space of families $(g(t))_{t \in I}$ of sections in the space of Riemannian metrics on $\mathbb{R}^{n}$ which are $C^{k}$ on $\mathbb{R}^{n} \times I$. Similarly, we define $\mathcal{M}^{\infty}, \mathcal{M}_{\text {loc }}^{k}$ and use $\mathcal{M}^{k}\left(\mathbb{R}^{n}\right)$ if the metric does not depend on $t$. We wish to point out that we use $C^{k}$ on non-compact sets to denote the space, where derivatives of order up to $k$ are in $L^{\infty}$. We also use $C_{\text {loc }}^{k}$.

Our existence result reads as follows.

Theorem 1.2. For every $\varepsilon>0$ there exists $0<\varepsilon_{0}(\varepsilon, n) \leq \varepsilon$, such that the following is true: if $g_{0} \in \mathcal{M}^{0}\left(\mathbb{R}^{n}\right)$ is a Riemannian metric on $\mathbb{R}^{n}$ which is $\varepsilon_{0}$-close to the standard metric $h=\delta$, then there exists a solution $g \in$ $\mathcal{M}^{\infty}\left(\mathbb{R}^{n},(0, \infty)\right) \cap \mathcal{M}_{\text {loc }}^{0}\left(\mathbb{R}^{n},[0, \infty)\right)$ to $(1.1)$ such that $g(t)$ is $\varepsilon$-close to $h$ for all $t$. As $t \rightarrow \infty$, the metrics $g(t)$ converge subsequentially in $\mathcal{M}_{\mathrm{loc}}^{\infty}\left(\mathbb{R}^{n}\right)$ to a complete flat metric.

In this situation, our main theorem addresses convergence to the Euclidean background metric.

Theorem 1.3. Let $g \in \mathcal{M}^{\infty}\left(\mathbb{R}^{n},(0, \infty)\right) \cap \mathcal{M}_{\mathrm{loc}}^{0}\left(\mathbb{R}^{n},[0, \infty)\right)$ be a solution to (1.1). Assume that $g(t)$ is $\tilde{\varepsilon}(n)$-close to the standard metric $h$ for all $t \geq 0$, for some $\tilde{\varepsilon}(n)$ chosen sufficiently small, and that $g_{0}$ is $\varepsilon(r)$-close to $h$ on $\mathbb{R}^{n} \backslash B_{r}(0)$ with $\varepsilon(r) \rightarrow 0$ as $r \rightarrow \infty$. Then

$$
g(t) \rightarrow h \quad \text { in } C^{\infty} \text { as } t \longrightarrow \infty
$$

That is,

$$
\sup _{\mathbb{R}^{n}}|g(t)-h| \longrightarrow 0
$$

as $t \rightarrow \infty$, and

$$
\sup _{\mathbb{R}^{n}}\left|{ }^{h} \nabla^{k} g(t)\right| \longrightarrow 0
$$

as $t \rightarrow \infty$ for all $k \in\{1,2, \ldots\}$. 
In this paper we will be concerned with the geometric quantities

$$
\varphi_{m}:=g^{a_{1} b_{1}} h_{b_{1} a_{2}} g^{a_{2} b_{2}} h_{b_{2} a_{3}} \cdots g^{a_{m} b_{m}} h_{b_{m} a_{1}}
$$

and

$$
\psi_{m}:=g_{a_{1} b_{1}} h^{b_{1} a_{2}} g_{a_{2} b_{2}} h^{b_{2} a_{3}} \cdots g_{a_{m} b_{m}} h^{b_{m} a_{1}} .
$$

We use the Einstein summation convention, i.e., we sum over repeated upper and lower indices like $a_{j}, b_{j}, 1 \leq j \leq m$, from 1 to $n$. If $\left(\lambda_{i}\right)$ are the eigenvalues of $\left(g_{i j}\right)$ with respect to $\left(h_{i j}\right)$, we get

$$
\varphi_{m}=\sum_{i=1}^{n} \frac{1}{\lambda_{i}^{m}} \quad \text { and } \quad \psi_{m}=\sum_{i=1}^{n} \lambda_{i}^{m}
$$

In particular

$$
\varphi_{m}+\psi_{m}-2 n=\sum_{i=1}^{n}\left(\frac{1}{\lambda_{i}^{m}}+\lambda_{i}^{m}-2\right)=\sum_{i=1}^{n} \frac{1}{\lambda_{i}^{m}}\left(\lambda_{i}^{m}-1\right)^{2} \geq 0
$$

is non-negative and vanishes precisely when $\lambda_{i}=1$ for all $i \in\{1, \ldots, n\}$.

Theorem 1.4. Let $n \geq 3$ and $g \in \mathcal{M}_{\mathrm{loc}}^{\infty}\left(\mathbb{R}^{n},[0, \infty)\right)$ be a solution to $(1.1)$, satisfying all the conditions of Theorem 1.3. Then there exist $m=m(n) \in \mathbb{N}$ and $\tilde{\varepsilon}(n)>0$, such that the following holds. If $1 \leq p<\frac{n}{2}$ can be chosen such that initially

$$
\int_{\mathbb{R}^{n}}\left(\varphi_{m}+\psi_{m}-2 n\right)^{p}<\infty
$$

or, equivalently,

$$
\|g(0)-h\|_{L^{2 p}}<\infty
$$

then there exists a smooth family $\left(\varphi_{t}\right)_{t \geq 0}$ of diffeomorphisms of $\mathbb{R}^{n}, \varphi_{0}=$ $\operatorname{id}_{\mathbb{R}^{\mathrm{n}}}$, such that for $\tilde{g}(t):=\varphi_{t}^{*} g(t)$ the family $(\tilde{g}(t))_{t \geq 0}$ is a smooth solution to the RF equation satisfying

$$
\tilde{g}(t) \rightarrow\left(\varphi_{\infty}\right)^{*} h \quad \text { in } \mathcal{M}^{\infty}\left(\mathbb{R}^{n}\right) \text { as } t \rightarrow \infty
$$

for some smooth diffeomorphism $\varphi_{\infty}$ of $\mathbb{R}^{n}$ which satisfies $\varphi_{t} \rightarrow \varphi_{\infty}$ in $C^{\infty}\left(\mathbb{R}^{n}, \mathbb{R}^{n}\right)$ as $t \rightarrow \infty$ and

$$
\left|\varphi_{\infty}(x)-x\right| \rightarrow 0 \quad \text { as }|x| \rightarrow \infty .
$$


Rewriting (1.5) in terms of a decay condition on $\varepsilon(r)$ in the closeness condition for $g_{0}$ (see Theorem 1.3), yields the following

Corollary 1.5. Let $n \geq 3$ and $g \in \mathcal{M}_{\mathrm{loc}}^{\infty}\left(\mathbb{R}^{n},[0, \infty)\right)$ be a solution to $(1.1)$, satisfying all the conditions of Theorem 1.3. If $g_{0}$ is $\varepsilon_{0}$-close to $h$ for some $\varepsilon_{0}=\varepsilon_{0}(n)>0$ sufficiently small, and in addition there exist constants $C>0$ and $\zeta>0$ such that $g_{0}$ is $\mathrm{Cr}^{-1-\zeta}$-close to $h$ on $\mathbb{R}^{n} \backslash B_{r}(0)$, then the same conclusions as in Theorem 1.4 hold.

Note that the solutions constructed in Theorem 1.2 satisfy the conditions of Theorem 1.3, but are not necessarily $\varepsilon(r)$-close to $h$ at infinity. We point out that these solutions may depend on the subsequence chosen in the construction in Section 4. Note, however, that it is not clear, whether solutions to the initial value problem (1.1) are unique, as in general $g_{0}$ does not have bounded curvature or a well-defined Riemannian curvature tensor. Even if $g(t)$ is $\varepsilon(n)$-close to $h$ on $\mathbb{R}^{n}$ for all $t>0$, we do not know, whether such solutions are unique. If we study only solutions as constructed in Section 4, we can replace the $\varepsilon(r)$-closeness condition imposed on $g_{0}$ by a considerably weaker integrability condition.

Theorem 1.6. Fix $p \geq 1, m=m(n) \in \mathbb{N}$ sufficiently large, and $\varepsilon_{0}=\varepsilon_{0}(n)$ $>0$ sufficiently small. Let $g_{0} \in \mathcal{M}^{0}\left(\mathbb{R}^{n}\right)$ be a Riemannian metric which is $\varepsilon_{0}$-close to the standard metric $h$. Let $g \in \mathcal{M}_{\mathrm{loc}}^{\infty}\left(\mathbb{R}^{n},(0, \infty)\right)$ be a solution to (1.1) as constructed in Section 4 . If for every $\delta>0$ the integral $I_{\delta}^{m, p}(0)$, as defined in Theorem 6.1, is finite, then

$$
g(t) \rightarrow h \quad \text { in } \mathcal{M}^{\infty}\left(\mathbb{R}^{n}\right) \text { as } t \rightarrow \infty
$$

In the situation of Theorem 1.6, if $I_{0}^{m, p}(0)$ is finite, we can show that the diffeomorphisms $\varphi_{t}$ stay bounded and converge in $C_{\mathrm{loc}}^{\infty}$ to a limiting diffeomorphism $\varphi_{\infty}$.

Outline. In order to prove stability of Euclidean $\mathbb{R}^{n}$ under the $h$-flow, we proceed as follows.

If we perturb the metric on $\mathbb{R}^{n}$, such that the perturbed metric is $C^{0}$ close to our original metric and the perturbation is small enough, this is preserved for all times. The perturbation is measured in terms of the deviation of the eigenvalues $\left(\lambda_{i}\right)$ of the perturbed metric with respect to the Euclidean metric from one. We have

$$
(1+\varepsilon)^{-1} \leq \lambda_{i} \leq 1+\varepsilon \quad \text { for } i=1, \ldots, n
$$


everywhere for all times and $0<\varepsilon=\varepsilon(n)$ provided that initially $\left(1+\varepsilon_{0}\right)^{-1}$ $\leq \lambda_{i} \leq 1+\varepsilon_{0}$ for some $0<\varepsilon_{0} \leq \varepsilon_{0}(n)$.

We have interior estimates for the gradient of the metric evolving under DeTurck flow. If we assume that $\lambda_{1} \leq \ldots \leq \lambda_{n}$, we get the estimate

$$
\left|\lambda_{i}(x, t)-\lambda_{i}(y, t)\right| \leq \frac{c}{\sqrt{t}} \cdot d_{\text {Eucl. }}(x, y) .
$$

Thus, for large times, the eigenvalues $\lambda_{i}$ are almost spatially constant in any Euclidean ball. It is, however, possible that these spatial constants vary in time. In particular, it is not clear up until now whether these constants converge as time tends to infinity.

Our perturbation of the Euclidean metric is small near infinity, i.e., all eigenvalues $\lambda_{i}$ approach one near infinity. Note, however, that we do not impose a decay rate on our initial metric, at which the eigenvalues converge to one as we approach spatial infinity. Still, we obtain for all positive times that the eigenvalues converge to one at spatial infinity.

In order to get uniform control on the eigenvalues, we use the quantity considered in (1.4) for some $m \in \mathbb{N}$. It vanishes precisely when $\lambda_{i}=1$ for all $i$ and measures the deviation from $\lambda_{i}=1$. The functions $\varphi_{m}$ and $\psi_{m}$ were initially considered by Shi in [12]. The key estimate is to show essentially that $\int\left(\varphi_{m}+\psi_{m}-2 n\right)$ is non-increasing in time. Here we integrate over the manifold at a fixed time. For technical reasons we have to consider a more complicated quantity. For details we refer to the respective proofs in Section 6 .

This implies convergence of the eigenvalues $\lambda_{i}(x, t)$ to 1 as $t \rightarrow \infty$, uniformly in $x$. For if this were not the case, we could pick points $\left(x_{k}, t_{k}\right)$ with $t_{k} \rightarrow \infty$ such that at least one $\lambda_{i}\left(x_{k}, t_{k}\right)$ differs significantly from 1 . If $t_{k}$ is large enough, we find a big ball, where at least one eigenvalue differs considerably from 1 . This yields an arbitrarily large contribution to $\int\left(\varphi_{m}+\psi_{m}-2 n\right)$ if $t_{k}$ is big compared to $\max _{i}\left|\lambda_{i}\left(x_{k}, t_{k}\right)-1\right|$, contradicting the monotonicity of $\int\left(\varphi_{m}+\psi_{m}-2 n\right)$. Thus $\lambda_{i}(x, t) \rightarrow 1$, uniformly in $x$ as $t \rightarrow \infty$, follows.

Remark 1.7. Note that all the above results also hold if we replace $\mathbb{R}^{n}$ by $\mathbb{T}^{k} \times \mathbb{R}^{n-k}$ for $n-k \geq 1$, where $\mathbb{T}^{k}$ is a flat $k$-dimensional torus.

In two space dimensions, Ricci flow is a conformal flow given by the evolution equation

$$
\frac{\partial}{\partial t} g=-R g
$$


where $R$ is the scalar curvature, see [6]. In this situation, we obtain (for details, see the appendix) the following.

Theorem 1.8. Let $g_{0}=e^{-u_{0}} h$, where $h=\delta$ is the standard Euclidean metric, and $u_{0} \in C^{0}\left(\mathbb{R}^{2}\right)$ such that

$$
\sup _{\mathbb{R}^{2} \backslash B_{r}(0)}|u| \rightarrow 0 \quad \text { as } r \rightarrow \infty \text {. }
$$

Then there exists a smooth solution $(g(t))_{t \in(0, \infty)}, g(t)=e^{-u(\cdot, t)} h$, to $R F$ such that $u(\cdot, t) \rightarrow u_{0}$ in $C_{\mathrm{loc}}^{0}$ as $t \searrow 0$. Furthermore, as $t \rightarrow \infty$, we obtain that $u(\cdot, t) \rightarrow 0$ in $C^{\infty}\left(\mathbb{R}^{2}\right)$.

For two-dimensional RF, Wu [16] studied long-time behavior and convergence of solutions. She studied initial complete metrics $g_{0}=e^{u_{0}} h$ which have bounded curvature and satisfy $e^{-u_{0}}\left|D u_{0}\right|^{2}<\infty$. In this case, she showed that a long-time solution to RF exists and that it "converges smoothly in the sense of modified subsequences" to a smooth limiting metric as $t \rightarrow \infty$. A smooth family of metrics $(g(t))_{t \in[0, \infty)}$ on $\mathbb{R}^{n}$ "converges smoothly in the sense of modified subsequences" to a metric $l$ on $\mathbb{R}^{n}$ as $t \rightarrow \infty$ if there exist diffeomorphisms $\varphi_{i}: \mathbb{R}^{n} \rightarrow \mathbb{R}^{n}$ and a sequence $t_{1}<t_{2}<\ldots$ with $t_{i} \rightarrow \infty$ as $i \rightarrow \infty$, such that $\left(\varphi_{i}\right)^{*}\left(g\left(t_{i}\right)\right) \rightarrow l$ smoothly on any fixed compact subset of $\mathbb{R}^{n}$. In particular, this does not imply uniform convergence on all of $\mathbb{R}^{n}$.

By imposing strong decay conditions on the curvature tensor at infinity, stability of flat space under RF was proved by Shi [13]. Stability of compact flat manifolds under RF was studied by Guenther et al. [4] and by Šešum [15]. In the rotationally symmetric situation stability of flat Euclidean space was investigated by Oliynyk and Woolgar [10]. Chau and the first author obtained a stability result for the Kähler potential of stationary rotationally symmetric solitons of positive holomorphic bisectional curvature under Kähler-Ricci flow [1]. Uniqueness of solutions to RF with bounded curvature on non-compact manifolds is discussed in $[2,8]$. Clutterbuck and the first two authors proved stability of convex rotationally symmetric translating solutions to mean curvature flow in [3]. Short-time existence results for $C^{0}$-metrics were shown in [14] using similar techniques to this paper.

The rest of the paper is organized as follows. We introduce our notation in Section 2 and recall some evolution equations in Section 3. In Section 4 we show the existence of solutions for all times. We prove interior closeness and a priori estimates in Section 5. In Section 6 we show that an integral quantity based on the expression in (1.4) is monotonically decreasing along the flow. Combining this with the interior gradient estimates, we obtain in 
Section 7 that the eigenvalues of $g_{i j}(x, t)$ with respect to the background metric converge uniformly to one as $t \rightarrow \infty$. An iteration scheme allows us to improve our gradient estimates for large times, see Section 8. In Section 9, we study the diffeomorphisms relating RF equation and (1.1), and show their convergence for large times. We address the proof of Theorem 1.6 in Section 10. Stability results for RF in two dimensions are addressed in the appendix.

\section{Notation}

Let $g=\left(g_{i j}\right)_{1 \leq i, j \leq n}$ be a Riemannian metric. By $(g(t))_{t \in(0, \infty)}$, we denote a family of metrics. We denote the inverse of $\left(g_{i j}\right)$ by $\left(g^{i j}\right)$ and use the Einstein summation convention for repeated upper and lower indices. If $g_{1}$ and $g_{2}$ are two metrics such that $\left(g_{1}\right)_{i j} \xi^{i} \xi^{j} \leq\left(g_{2}\right)_{i j} \xi^{i} \xi^{j}$ for all $\left(\xi^{i}\right) \in \mathbb{R}^{n}$, we denote this by $g_{1} \leq g_{2}$. Unless otherwise stated, geometric quantities like covariant derivatives $\nabla_{i}$ and Christoffel symbols $\Gamma_{i j}^{k}$ are computed with respect to the evolving metrics $g(t)$. We use indices $h$ to denote quantities depending on the metric $h$, e.g., ${ }^{h} \nabla g$ denotes the covariant derivative of $g$ with respect to $h$, i.e., a partial derivative. In short formulae, we also use $\nabla g$ instead. With the exception of the beginning of Section $4, h$ will always denote the standard metric on $\mathbb{R}^{n}$. On $\mathbb{R}^{n}$, we will always use co-ordinates such that $h_{i j}=\delta_{i j}$. A ball of radius $r$, centered at $x$, is denoted by $B_{r}(x)$. We will only use Euclidean balls. The norm ${ }^{h}|\cdot|$ is computed with respect to the flat background metric $h$. We also denote it by $|\cdot|$. The letter $c$ denotes generic constants.

In formulae, where we use $\lambda_{i}$ to denote the eigenvalues of $g_{i j}$ with respect to $h_{i j}$, we will always assume that $h_{i j}=\delta_{i j}$ in the co-ordinate system chosen. Several times, we will use that ${ }^{h}\left|g_{i j}(x, t)-g_{i j}(y, t)\right| \leq A$ implies that $\left|\lambda_{i}(x, t)-\lambda_{i}(y, t)\right| \leq A$ if we assume that $\lambda_{1}(\cdot, t) \leq \ldots \leq \lambda_{n}(\cdot, t)$. Similarly, ${ }^{h}\left|g_{i j}(x, t)-h_{i j}\right| \leq \bar{A}$ implies $\left|\lambda_{i}(x, t)-1\right| \leq A$.

\section{Evolution equations}

In this section, we collect some evolution equations from [12] and state some direct consequences.

Assume that in appropriate coordinates, we have at a fixed point and at a fixed time $h_{i j}=\delta_{i j}, g_{i j}=\operatorname{diag}\left(\lambda_{1}, \lambda_{2}, \ldots, \lambda_{n}\right), \lambda_{i}>0$. 
The evolution equation for the metric, computed with respect to a flat background metric, is [12, Lemma 2.1],

$$
\begin{aligned}
\frac{\partial}{\partial t} g_{i j}= & g^{a b^{h}} \nabla_{a}{ }^{h} \nabla_{b} g_{i j} \\
& +\frac{1}{2} g^{a b} g^{p q}\left({ }^{h} \nabla_{i} g_{p a}{ }^{h} \nabla_{j} g_{q b}+2^{h} \nabla_{a} g_{j p}{ }^{h} \nabla_{q} g_{i b}-2{ }^{h} \nabla_{a} g_{j p}{ }^{h} \nabla_{b} g_{i q}\right. \\
& \left.-2^{h} \nabla_{j} g_{p a}{ }^{h} \nabla_{b} g_{i q}-2^{h} \nabla_{i} g_{p a}{ }^{h} \nabla_{b} g_{j q}\right) .
\end{aligned}
$$

Consider $\varphi_{m}$ as defined in (1.2). The evolution equation of $\varphi_{m}$ for a flat background metric $h_{i j}$ is [12, Lemma 2.2, Equation (70)],

$$
\begin{aligned}
\frac{\partial}{\partial t} \varphi_{m}= & g^{a b^{h}} \nabla_{a}{ }^{h} \nabla_{b} \varphi_{m} \\
& -m \sum_{i, j, a} \frac{1}{\lambda_{a}}\left[\sum_{k=2}^{m}\left(\frac{1}{\lambda_{i}}\right)^{k}\left(\frac{1}{\lambda_{j}}\right)^{m+2-k}\right]\left({ }^{h} \nabla_{a} g_{i j}\right)^{2} \\
& -\sum_{i, q, k} \frac{m}{2 \lambda_{i}^{m+1} \lambda_{q} \lambda_{k}}\left({ }^{h} \nabla_{k} g_{i q}+{ }^{h} \nabla_{q} g_{i k}-{ }^{h} \nabla_{i} g_{q k}\right)^{2} .
\end{aligned}
$$

We deduce for $g(t)$ that are $\varepsilon$-close to $h$ that

$$
\frac{\partial}{\partial t} \varphi_{m} \leq g^{i j^{h}} \nabla_{i}^{h} \nabla_{j} \varphi_{m}-m(m-1)(1+\varepsilon)^{-(m+3)} \sum_{i, j, k}\left({ }^{h} \nabla_{k} g_{i j}\right)^{2}
$$

From the evolution equation of the metric, we get for $\psi_{m}$, as introduced in (1.3), the evolution equation

$$
\begin{aligned}
& \frac{\partial}{\partial t} \psi_{m}=m h^{a_{1} b_{1}} g_{b_{1} a_{2}} h^{a_{2} b_{2}} g_{b_{2} a_{3}} \cdots h^{a_{m} b_{m}} \frac{\partial}{\partial t} g_{b_{m} a_{1}} \\
& =m h^{a_{1} b_{1}} g_{b_{1} a_{2}} h^{a_{2} b_{2}} g_{b_{2} a_{3}} \cdots h^{a_{m} b_{m}}\left(g^{i j^{h}} \nabla_{i}{ }^{h} \nabla_{j} g_{b_{m} a_{1}}\right) \\
& +c(n) m \underbrace{h^{-1} * \cdots * h^{-1}}_{m} * \underbrace{g * \cdots * g}_{m-1} * g^{-1} * g^{-1} *{ }^{h} \nabla g *{ }^{h} \nabla g \\
& =g^{i j^{h}} \nabla_{i}{ }^{h} \nabla_{j} \psi_{m}-m \sum_{i, j, a}\left[\sum_{k=0}^{m-2} \lambda_{i}^{k} \lambda_{j}^{m-2-k}\right] \frac{1}{\lambda_{a}}\left({ }^{h} \nabla_{a} g_{i j}\right)^{2} \\
& +c(n) m \underbrace{h^{-1} * \cdots * h^{-1}}_{m} * \underbrace{g * \cdots * g}_{m-1} * g^{-1} * g^{-1} *{ }^{h} \nabla g *{ }^{h} \nabla g \text {, }
\end{aligned}
$$


where $*$ indicates contractions and linear combinations of contractions. The factor $c(n)$ indicates that the number of the respective terms depends only on $n$. Therefore, we deduce that

$$
\begin{aligned}
\frac{\partial}{\partial t} \psi_{m} \leq & g^{i j^{h}} \nabla_{i}^{h} \nabla_{j} \psi_{m} \\
& -m(m-1)(1+\varepsilon)^{-(m-1)} \sum_{i, j, k}\left({ }^{h} \nabla_{k} g_{i j}\right)^{2} \\
& +c(n) m(1+\varepsilon)^{m+1} \sum_{i, j, k}\left({ }^{h} \nabla_{k} g_{i j}\right)^{2} .
\end{aligned}
$$

\section{Existence}

In this section we prove long-time existence of the $h$-flow for a smooth initial metric $g_{0}$ which is $\varepsilon$-close to $h=\delta$. Short-time existence for Ricci-DeTurck flow on non-compact manifolds was first proved in [12]. Short-time existence for an arbitrary smooth background metric $h$ with bounded curvature, when $g_{0}$ is $\varepsilon$-close to $h$, was proved in [14], using similar techniques to those of Shi. We also reprove the short-time existence here for completeness. (Note that some of the citations in [14] in the proof of the short-time existence were incorrect.)

Lemma 4.1. Let $(g(t))_{t \in[0, T)}$ be a smooth $\varepsilon$-close solution to the $h$-flow with flat background metric $h=\delta$ on a closed ball $D \subset \mathbb{R}^{n}$ with $0<\varepsilon \leq \varepsilon(n)$ and $\left.g(\cdot, t)\right|_{\partial D}=\left.h(\cdot)\right|_{\partial D}$. Then

$$
\sup _{D \times[0, T)}\left|{ }^{h} \nabla^{m} g\right|^{2} \leq c(n, m, D, T, g(0)) .
$$

Proof. We proceed exactly as in the proof of Shi [12, Lemma 3.1]: where he uses $\tilde{g}$, we use $h$. The only other minor difference is that the dependence on $g(0)$ does not explicitly appear in Shi's paper [12, Lemma 3.1]. This is because he has $\tilde{g}=g(0)$.

Lemma 4.2. Let $(g(t))_{t \in[0, T)}$ be a smooth solution to the h-flow on $B_{i}$, $i \in \mathbb{N}$, where $h$ is the standard metric on $\mathbb{R}^{n}$. Then for all $\varepsilon>0$ there exists 
$0<\varepsilon_{0}(n, \varepsilon)<\varepsilon$, such that the following holds. If $g(0)$ is $\varepsilon_{0}$-close to $h$ and $g(t)=h$ on $\partial B_{i} \times[0, T)$, then $g(t)$ is $\varepsilon$-close to $h$ for all $t \in[0, T)$.

Proof. Consider $\Phi:=\varphi_{m}+\psi_{m}-2 n$ as in (1.4). It vanishes on $\partial D \times[0, T)$. The evolution equations in Section 3 imply that

$$
\frac{\partial}{\partial t} \Phi \leq g^{i j^{h}} \nabla_{i}^{h} \nabla_{j} \Phi
$$

as long as $g(t)$ is $\tilde{\varepsilon}$-close to $h$ for $\tilde{\varepsilon}>0$ satisfying

$$
c(n)(1+\tilde{\varepsilon})^{2 m} \leq(m-1) .
$$

Choose $\tilde{\varepsilon}=\tilde{\varepsilon}(n)$ and $m=m(n)$ accordingly.

Fix $\delta>0$ so that $\Phi \leq 2 \delta$ implies, see (1.4), that the metric is $\varepsilon$-close to $h$, in particular $\tilde{\varepsilon}$-close to $h$. Now fix $\varepsilon_{0}=\varepsilon_{0}(n)>0$ such that $\varepsilon_{0}<\varepsilon$ and so that $\Phi \leq \delta$ for every metric which is $\varepsilon_{0}$-close to $h$.

Consider the maximal time interval $I \subset[0, T)$ on which $\Phi \leq 2 \delta$. We may assume that $I=[0, \tau]$ with $\tau<T$. This implies that $(g(t))_{t \in[0, \tau]}$ is $\tilde{\varepsilon}$-close to $h$ (even $\varepsilon$-close to $h$ ). According to (4.1) and the maximum principle, $\max _{x \in D} \Phi(x, t)$ is non-increasing in $t$ for all $t \in[0, \tau]$. Thus $\Phi(\tau) \leq \Phi(0) \leq \delta$. This contradicts the choice of $\tau$. Therefore $I=[0, T)$ and $(g(t))_{t \in[0, T)}$ is $\varepsilon$-close to $h$.

In the remainder of this section, we will denote with $\varepsilon_{0}(n, \varepsilon)>0$ the constant in Lemma 4.2. Note that although we allow for arbitrary $\varepsilon>0$, it follows from the proof that $\varepsilon_{0}(n, \varepsilon) \leq \bar{\varepsilon}$ for some small $\bar{\varepsilon}=\bar{\varepsilon}(n)>0$.

Theorem 4.3. Let $\varepsilon>0$ be given, and $g_{0}$ be smooth and $\varepsilon_{0}(\varepsilon, n)$-close to $h=\delta$ on a closed ball $D \subset \mathbb{R}^{n}$. Assume that $g_{0}=h$ near $\partial D$. Then there exists a unique smooth solution $(g(t))_{t \in[0, \infty)}$ to the $h$-flow with $g(0)=g_{0}$ and $\left.g(t)\right|_{\partial D}=\left.h\right|_{\partial D}$. Furthermore, $(g(t))_{t \in[0, \infty)}$ is $\varepsilon$-close to $h$.

Proof. Lemma 4.2 implies that a prospective solution $g(t)$ is $\varepsilon$-close to $h$ as long as it exists. Therefore, we can apply the a priori bounds of Lemma 4.1 on a bounded time interval. We may then use the same arguments as in $[9$, Theorem 7.1 , Chapter VII $]$ to show that a smooth solution exists (the argument used there is based on the Leray-Schauder fixed point argument of $[9$, Theorem 6.1, Chapter V]). Note that this argument works for every finite time interval. 
Theorem 4.4. Let $g \in \mathcal{M}^{\infty}\left(B_{R},[0, T]\right)$ be $\tilde{\varepsilon}$-close to $h$, solving (1.1), where $\tilde{\varepsilon}=\tilde{\varepsilon}(n)>0$ is sufficiently small and $h=\delta$. Then

$$
\sup _{B_{R / 2} \times[0, T]}\left|{ }^{n} \nabla^{m} g\right|^{2} \leq c\left(m, n,\left.g(0)\right|_{B_{R}}, T\right) .
$$

Proof. This follows using the arguments of Shi [12, Lemmas 4.1 and 4.2].

Theorem 4.5. Let $\varepsilon>0$ be given, and $g_{0}$ be smooth and $\varepsilon_{0}(n, \varepsilon)$-close to the standard Riemannian metric $h=\delta$ on $\mathbb{R}^{n}$. Then there exists a $\varepsilon$-close solution $g \in \mathcal{M}_{\mathrm{loc}}^{\infty}\left(\mathbb{R}^{n},[0, \infty)\right)$ to the $h$-flow (1.1).

Proof. Let $B_{i}$ be the balls of radius $i$ and center 0 (with respect to the Euclidean metric). Set ${ }^{i} g_{0}=\eta_{i} g_{0}+\left(1-\eta_{i}\right) h$, where $\eta_{i}: \mathbb{R}^{n} \rightarrow \mathbb{R}$ is smooth, and satisfies $\eta_{i}=0$ on $B_{i}-B_{i-1}, \eta_{i}=1$ on $B_{i-2}, 0 \leq \eta \leq 1$ and $\left|\nabla^{m} \eta_{i}\right|^{2} \leq$ $c(m, n)$. Note that ${ }^{i} g_{0}$ is $\varepsilon_{0}$-close to $h$. Let ${ }^{i} g(t) \in \mathcal{M}^{\infty}\left(B_{i},[0, \infty)\right)$ be the solution coming from the local existence theorem (Theorem 4.3) above. Using the interior estimates of Theorem 4.4 above, we see that the solutions all satisfy

$$
\sup _{B_{j} \times[0, T]}\left|\nabla^{m}\left(\frac{d}{d t}\right)^{k}\left({ }^{i} g\right)\right|^{2} \leq c\left(j,\left.g_{0}\right|_{B_{2 j}}, m, k, n, T\right),
$$

for all $i$ big enough, and so, using a diagonal subsequence argument, we can find a subsequence which converges to a solution $g \in \mathcal{M}_{\mathrm{loc}}^{\infty}\left(\mathbb{R}^{n},[0, \infty)\right)$ where the convergence ${ }^{i} g \rightarrow g$ is uniform on $B_{j} \times[0, T]$ for every fixed $j \in \mathbb{N}$ and $T \in(0, \infty)$ and $g(0)=g_{0}$.

We do not know, whether the solution obtained in Theorem 4.5 is unique or depends on the subsequence chosen.

\section{Interior estimates}

Note that the following lemma does not imply $\lambda_{i}(x, t)-1 \rightarrow 0$ for $|x| \rightarrow \infty$ uniformly in $t$, i.e.,

$$
\lim _{R \rightarrow \infty} \sup _{x \in \mathbb{R}^{n} \backslash B_{R}} \sup _{i \in\{1, \ldots, n\}} \sup _{t \in[0, \infty)}\left|\lambda_{i}(x, t)-1\right|
$$

may be non-zero. 
Lemma 5.1. Let $g \in \mathcal{M}_{\mathrm{loc}}^{\infty}\left(\mathbb{R}^{n},(0, \infty)\right)$ be a solution to (1.1) with $g_{0}$ as in Theorem 1.3. Then the eigenvalues $\left(\lambda_{i}(x, t)\right)$ of $\left(g_{i j}(x, t)\right)$ with respect to $\left(h_{i j}(x)\right)$ uniformly tend to 1 in bounded time intervals,

$$
\lim _{|x| \rightarrow \infty} \lambda_{i}(x, t)=1
$$

This lemma is a direct consequence of the following interior closeness estimate.

Lemma 5.2. Let $g \in \mathcal{M}_{\mathrm{loc}}^{\infty}\left(\mathbb{R}^{n},(0, \infty)\right)$ be a solution to $(1.1)$ which is $\tilde{\varepsilon}(n)$ close to the Euclidean background metric h. Assume that $g_{0}$ is $\varepsilon$-close to $h$ on some ball $B_{R}\left(x_{0}\right)$ for some $\varepsilon \leq \tilde{\varepsilon}(n) / 2$. Then there is a constant $\gamma(n)>0$ such that $g(t)$ is $2 \varepsilon$-close to $h$ on $B_{R / 2}\left(x_{0}\right)$ for $t \in\left[0, \gamma R^{2}\right]$.

Proof. According to the estimates in Section 3, there exists an $\varepsilon(n)>0$ and $m(n) \in \mathbb{N}$ such that

$$
\frac{\partial}{\partial t}\left(\varphi_{m}+\psi_{m}-2 n\right) \leq g^{a b^{h}} \nabla_{a}{ }^{h} \nabla_{b}\left(\varphi_{m}+\psi_{m}-2 n\right)-\sum_{i, j, k}\left({ }^{h} \nabla_{k} g_{i j}\right)^{2} .
$$

If the background metric $h$ is the standard flat metric on $\mathbb{R}^{n}$, we define for $\lambda>0$

$$
g_{i j}^{\lambda}(x, t)=g_{i j}\left(\frac{x}{\lambda}, \frac{t}{\lambda^{2}}\right)
$$

If $g$ is a solution to the $h$-flow, $g^{\lambda}$ is also a solution to the $h$-flow as $h$ is the standard metric on $\mathbb{R}^{n}$. Moreover, $g^{\lambda}$ is $\varepsilon$-close to $h$, if $g$ is $\varepsilon$-close to $h$.

By scaling with the factor $\lambda=1 / R$ and translation we can assume that $R=1$ and $x_{0}=0$. Let $\eta \in C_{c}^{\infty}\left(B_{1}\right)$ be such that $0 \leq \eta \leq 1$ and $\eta \equiv 1$ on $B_{1 / 2}$ and let

$$
\zeta:=\eta \cdot\left(\varphi_{m}+\psi_{m}-2 n\right)
$$

We compute

$$
\begin{aligned}
\frac{\partial}{\partial t} \zeta \leq & g^{a b^{h}} \nabla_{a}{ }^{h} \nabla_{b} \zeta-2 g^{a b^{h}} \nabla_{a} \eta^{h} \nabla_{b}\left(\varphi_{m}+\psi_{m}-2 n\right) \\
& -\left(\varphi_{m}+\psi_{m}-2 n\right) g^{a b^{h}} \nabla_{a}{ }^{h} \nabla_{b} \eta-\eta \sum_{i, j, k}\left({ }^{h} \nabla_{k} g_{i j}\right)^{2} \\
\leq & g^{a b^{h}} \nabla_{a}{ }^{h} \nabla_{b} \zeta+c
\end{aligned}
$$


since

$$
\left|{ }^{h} \nabla\left(\varphi_{m}+\psi_{m}-2 n\right)\right|^{2} \leq c \sum_{i, j, k}\left({ }^{h} \nabla_{k} g_{i j}\right)^{2} \quad \text { and } \quad|\nabla \eta|^{2} / \eta \leq C\left(n,\|\eta\|_{C^{2}}\right) .
$$

So by the maximum principle the maximum of $\zeta$ grows at most linearly, which implies the stated estimate.

By scaling we want to extend a priori derivative estimates for the metric to balls of any radius. If the background metric $h$ is not necessarily flat, the third author [14] obtained the following

Theorem 5.3. Let $R>0$. Let $h$ be a complete background metric of bounded curvature. Fix $T=T(n, R, h)>0$ sufficiently small. Fix a point $x_{0}$. Let $g$ be a solution to the $h$-flow on ${ }^{h} B_{R}\left(x_{0}\right) \times(0, T)$ which is $\tilde{\varepsilon}$-close to the background metric $h$ for $\tilde{\varepsilon}=\tilde{\varepsilon}(n)>0$ fixed sufficiently small, where ${ }^{h} B_{R}\left(x_{0}\right)$ denotes a geodesic ball of radius $R$ with respect to the metric $h$. Then

$$
\left|\nabla^{h} g(x, t)\right| \leq \frac{c(n, i, R)}{t^{i / 2}}
$$

for all $(x, t) \in{ }^{h} B_{R / 2}\left(x_{0}\right) \times(0, T)$ and all $i \in \mathbb{N}$.

Applying Theorem 5.3 to $g^{\lambda}$ as defined in the proof of Lemma 5.2, we get directly the following.

Corollary 5.4. Let $h$ denote the standard flat metric on $\mathbb{R}^{n}$ and assume that $\varepsilon=\varepsilon(n)>0$ and $\gamma=\gamma(n)>0$ are chosen sufficiently small. If $g$ solves the $h$-flow in $B_{R}\left(x_{0}\right) \times\left(0, \gamma R^{2}\right)$ for some $x_{0} \in \mathbb{R}^{n}, R>0$, and if $g$ is $\varepsilon$-close to $h$ on $B_{R}\left(x_{0}\right) \times\left(0, \gamma R^{2}\right)$, then we get the a priori estimates

$$
\left|{ }^{h} \nabla^{i} g(x, t)\right| \leq \frac{c(n, i)}{t^{i / 2}}
$$

for all $(x, t) \in B_{R / 2}\left(x_{0}\right) \times\left(0, \gamma R^{2}\right)$ and all $i \in \mathbb{N}$. We set $c_{1}:=c(n, 1)$.

Proof of Theorem 1.2. Let us first assume that $g_{0} \in \mathcal{M}_{\mathrm{loc}}^{\infty}\left(\mathbb{R}^{n}\right)$. Theorem 4.5 implies that an $\varepsilon$-close solution $g \in \mathcal{M}_{\mathrm{loc}}^{\infty}\left(\mathbb{R}^{n},[0, \infty)\right)$ to $(1.1)$ exists. The interior decay estimates of Corollary 5.4 and Arzelà-Ascoli imply that the metrics $g(t)$ converge subsequentially in $\mathcal{M}_{\text {loc }}^{\infty}$ to a complete flat metric as $t \rightarrow \infty$.

We approximate $g_{0} \in \mathcal{M}_{0}\left(\mathbb{R}^{n}\right)$ by smooth metrics ${ }^{i} g_{0}$ preserving the $\varepsilon_{0}$-closeness. Thus we obtain solutions ${ }^{i} g \in \mathcal{M}_{\text {loc }}^{\infty}$ which are $\varepsilon$-close to $h$. 
In view of the interior a priori estimates we obtain a limiting solution $g \in$ $\mathcal{M}_{\mathrm{loc}}^{\infty}\left(\mathbb{R}^{n},(0, \infty)\right)$.

Note that $g(t) \rightarrow g_{0}$ in $\mathcal{M}_{\text {loc }}^{0}$ as $t \searrow 0$ : fix a point $x_{0} \in \mathbb{R}^{n}$ and use $g_{0}\left(x_{0}\right)$ as a flat background metric on $\mathbb{R}^{n}$. Thus the metrics ${ }^{i} g$ are again solutions to the $h$-flow with $h=g_{0}\left(x_{0}\right)$. Hence we can apply the interior closeness estimates of Lemma 5.2 in order to see that all metrics ${ }^{i} g$ attain their initial values uniformly in $i$. This implies that $g(t) \rightarrow g_{0}$ as $t \searrow 0$ locally uniformly.

\section{Integral estimates}

In this section, we are once again concerned with the quantity $\varphi_{m}+\psi_{m}-2 n$ as introduced in (1.4).

Theorem 6.1. Fix $\delta>0, \quad m=m(n) \in \mathbb{N}$ and $p \geq 1$. Let $g \in \mathcal{M}_{\mathrm{loc}}^{\infty}$ $\left(\mathbb{R}^{n},(0, \infty)\right)$ be a solution to (1.1) which is $\tilde{\varepsilon}(n, m)$-close to the standard Euclidean metric $h$ for some $1 \geq \tilde{\varepsilon}=\tilde{\varepsilon}(n, m)>0$ sufficiently small. Let $g_{0}$ be as in Theorem 1.3. Then the integral

$$
I(t) \equiv I_{\delta}^{m, p}(t):=\frac{1}{p} \int_{\mathbb{R}^{n}}\left(\varphi_{m}+\psi_{m}-2 n-\delta\right)_{+}^{p} d x \equiv \frac{1}{p} \int_{\mathbb{R}^{n}} \Phi_{m, \delta}^{p}
$$

is non-increasing in time.

Proof. As $g$ is $\tilde{\varepsilon}$-close to $h, \varphi_{m}+\psi_{m}-2 n$ is uniformly bounded above. According to Lemma 5.1, we see that $\varphi_{m}+\psi_{m}-2 n \rightarrow 0$ for $|x| \rightarrow \infty$ and $t$ bounded above. Thus the positive part of $\varphi_{m}+\psi_{m}-2 n-\delta$ is contained in a compact set for every bounded time interval and $I(t)$ is finite there.

Assume that in appropriate co-ordinates, we have at a fixed point $h_{i j}=$ $\delta_{i j}, g_{i j}=\operatorname{diag}\left(\lambda_{1}, \lambda_{2}, \ldots, \lambda_{n}\right), \lambda_{i}>0$.

Recall from Section 3 that we can estimate in the evolution equation of $\varphi_{m}+\psi_{m}$ for metrics $\varepsilon$-close to $h$ as follows

$$
\begin{aligned}
\frac{\partial}{\partial t}\left(\varphi_{m}+\psi_{m}\right) \leq & g^{i j^{h}} \nabla_{i}{ }^{h} \nabla_{j}\left(\varphi_{m}+\psi_{m}\right) \\
& -m(m-1)(1+\varepsilon)^{-(m-1)} \sum_{i, j, k}\left({ }^{h} \nabla_{k} g_{i j}\right)^{2} \\
& +c(n) m(1+\varepsilon)^{m+1} \sum_{i, j, k}\left({ }^{h} \nabla_{k} g_{i j}\right)^{2} .
\end{aligned}
$$


We now want to show that $I(t)$ is decreasing in time for $m$ and $\varepsilon$ chosen properly. Note that $\Phi_{m, \delta}^{p}$ is Lipschitz continuous in space and time, and the support of $\Phi_{m, \delta}^{p}$ is contained in $B_{R}(0)$ for some $R>0$ on bounded time intervals. This yields that $I(t)$ is Lipschitz continuous in time as well and thus also absolutely continuous. By Sard's theorem we know that for almost every $\delta>0$ the sets

$$
U_{\delta} \equiv U_{\delta}(t):=\left\{x \in \mathbb{R}^{n} \mid \varphi_{m}(x, t)+\psi_{m}(x, t)-2 n>\delta\right\}
$$

have a smooth boundary for almost every $t$. For such a $\delta$ and $0 \leq t_{1}<t_{2}$, we can compute

$$
\begin{aligned}
I\left(t_{2}\right)-I\left(t_{1}\right) & =\int_{t_{1}}^{t_{2}} \frac{1}{p} \frac{d}{d \tau} \int_{\mathbb{R}^{n}} \Phi_{m, \delta}^{p} d x d \tau \\
& =\int_{t_{1}}^{t_{2}} \int_{U_{\delta}} \Phi_{m, \delta}^{p-1} \frac{\partial}{\partial \tau}\left(\varphi_{m}+\psi_{m}\right) d x d \tau
\end{aligned}
$$

as $\Phi_{m, \delta}$ is compactly supported as long as $t$ is finite. Since the boundary of $U_{\delta}$ is smooth we may estimate and integrate by parts

$$
\begin{aligned}
I\left(t_{2}\right)-I\left(t_{1}\right) \leq & \int_{t_{1}}^{t_{2}} \int_{U_{\delta}} \Phi_{m, \delta}^{p-1}\left(g^{i j^{h}} \nabla_{i}{ }^{h} \nabla_{j}\left(\varphi_{m}+\psi_{m}\right)\right) d x d \tau \\
& -\int_{t_{1}}^{t_{2}} \int_{U_{\delta}} m(m-1)(1+\varepsilon)^{-(m-1)} \Phi_{m, \delta}^{p-1} \sum_{i, j, k}\left({ }^{h} \nabla_{k} g_{i j}\right)^{2} d x d \tau \\
& +\int_{t_{1}}^{t_{2}} \int_{U_{\delta}} c(n) m(1+\varepsilon)^{m+1} \Phi_{m, \delta}^{p-1} \sum_{i, j, k}\left({ }^{h} \nabla_{k} g_{i j}\right)^{2} d x d \tau \\
= & \int_{t_{1}}^{t_{2}} \int_{\partial U_{\delta}} \Phi_{m, \delta}^{p-1} \nu_{i} g^{i j^{h}} \nabla_{j} \Phi_{m, \delta} d \sigma d t \\
& -\int_{t_{1}}^{t_{2}} \int_{U_{\delta}}(p-1) \Phi_{m, \delta}^{p-2} g^{i j h} \nabla_{i} \Phi_{m, \delta}{ }^{h} \nabla_{j} \Phi_{m, \delta} d x d \tau \\
& -\int_{t_{1}}^{t_{2}} \int_{U_{\delta}} \Phi_{m, \delta}^{p-1}\left({ }^{h} \nabla_{i} g^{i j}\right)^{h} \nabla_{j} \Phi_{m, \delta} d x d \tau \\
& +\int_{t_{1}}^{t_{2}} \int_{U_{\delta}}(-m(m-1))(1+\varepsilon)^{-m+1} \Phi_{m, \delta}^{p-1} \sum_{i, j, k}\left({ }^{h} \nabla_{k} g_{i j}\right)^{2} d x d \tau \\
& +\int_{t_{1}}^{t_{2}} \int_{U_{\delta}} c(n) m(1+\varepsilon)^{m+1} \Phi_{m, \delta}^{p-1} \sum_{i, j, k}\left({ }^{h} \nabla_{k} g_{i j}\right)^{2} d x d \tau
\end{aligned}
$$




$$
\begin{aligned}
\leq & m \int_{t_{1}}^{t_{2}} \int_{U_{\delta}} \Phi_{m, \delta}^{p-1} \sum_{i, j, k}\left({ }^{h} \nabla_{k} g_{i j}\right)^{2} \\
& \cdot\left(-(m-1)(1+\varepsilon)^{-m+1}+c(n)(1+\varepsilon)^{m+3}\right) d x d \tau
\end{aligned}
$$

where we used generic constants $c(n)$, the estimates

$$
\begin{aligned}
& { }^{h}\left|{ }^{h} \nabla g^{i j}\right| \leq c(1+\varepsilon)^{2} \cdot \sqrt{\sum_{i, j, k}\left({ }^{h} \nabla_{k} g_{i j}\right)^{2}}, \\
& { }^{h}\left|{ }^{h} \nabla \Phi_{m, \delta}\right| \leq c m(1+\varepsilon)^{m+1} \cdot \sqrt{\sum_{i, j, k}\left({ }^{h} \nabla_{k} g_{i j}\right)^{2}}
\end{aligned}
$$

and the fact that ${ }^{h} \nabla \Phi_{m, \delta}$ is antiparallel to the outer unit normal $\nu$ of $U_{\delta}$ along $\partial U_{\delta}$. Note that the integration by parts involving the exponent $p-2$ above is justified by applying the divergence theorem on sets $U_{\delta_{i}}$ for a sequence $\delta_{i} \searrow \delta$, where we can assume by Sard's theorem that $\partial U_{\delta_{i}}$ is smooth for all $i$ and that $\partial U_{\delta_{i}} \rightarrow \partial U_{\delta}$. If $m$ and $\varepsilon$ are such that

$$
1+c(n)(1+\varepsilon)^{2 m+2} \leq m
$$

we obtain that the right-hand side is non-positive and our theorem follows for such a $\delta$. Fix $\tilde{\varepsilon}>0$ accordingly. Since

$$
I_{m, p}^{\delta_{i}}(t) \rightarrow I_{m, p}^{\delta}(t)
$$

for $\delta_{i} \rightarrow \delta$ we obtain the above monotonicity for all $\delta$.

Corollary 6.2. Let $g \in \mathcal{M}_{\mathrm{loc}}^{\infty}\left(\mathbb{R}^{n},(0, \infty)\right)$ be a solution to $(1.1)$ with $g_{0}$ as in Theorem 1.3. Let $m, p$ and $\tilde{\varepsilon}$ be as in Theorem 6.1. If $I_{0}^{m, p}(0)$ is finite, then $I_{0}^{m, p}(t)$ is non-increasing and thus

$$
I^{m, p}(t):=\frac{1}{p} \int_{\mathbb{R}^{n}}\left(\varphi_{m}+\psi_{m}-2 n\right)^{p} d x=I_{0}^{m, p}(t) \leq I_{0}^{m, p}(0)<\infty .
$$

Proof. Theorem 6.1 implies that

$$
I_{\delta}^{m, p}(t) \leq I_{\delta}^{m, p}(0) \leq I_{0}^{m, p}(0)
$$

for every $\delta>0$. For $\delta \searrow 0$, we get

$$
I_{\delta}^{m, p}(t) \rightarrow I_{0}^{m, p}(t)
$$

and our claim follows. 


\section{Convergence of eigenvalues}

Lemma 7.1. Let $g \in \mathcal{M}_{\mathrm{loc}}^{\infty}\left(\mathbb{R}^{n},(0, \infty)\right)$ be a solution to $(1.1)$ with $g_{0}$ as in Theorem 1.3, such that $g(t)$ is $\tilde{\varepsilon}(n)$-close to $h$ for all $t$. Then the eigenvalues $\left(\lambda_{i}\right)_{1 \leq i \leq n}$ of $g(t)$ with respect to the background metric $h$ converge uniformly to one as $t \rightarrow \infty$,

$$
\sup _{x \in \mathbb{R}^{n}}\left|\lambda_{i}(x, t)-1\right| \rightarrow 0 \quad \text { as } t \rightarrow \infty
$$

Proof. Denote the eigenvalues of $g_{i j}$ with respect to $h_{i j}$ by $\left(\lambda_{i}\right)$. It is convenient to assume that $\lambda_{1} \leq \ldots \leq \lambda_{n}$.

Assume that the lemma were false. Then we could find $\zeta>0$ and a sequence $\left(x_{k}, t_{k}\right)_{k \in \mathbb{N}}$ in $\mathbb{R}^{n} \times[0, \infty)$ with $t_{k} \rightarrow \infty$ so that

$$
\max _{i \in\{1, \ldots, n\}}\left|\lambda_{i}\left(x_{k}, t_{k}\right)-1\right| \geq 2 \zeta
$$

The strategy of the proof is to use Corollary 5.4 with $i=1$ to show that we find a controlled spatial neighborhood of $\left(x_{k}, t_{k}\right)$ such that (7.1) is fulfilled with $2 \zeta$ replaced by $\zeta$, i.e., the eigenvalues are not all close to one there. Therefore, $\varphi_{m}+\psi_{m}-2 n$ is estimated from below by a positive constant in that neighborhood. For large values of $t$, these neighborhoods can be chosen arbitrarily large. Thus $I_{\delta}^{m, p}$ becomes large, if $\delta$ is chosen small enough. This contradicts Theorem 6.1.

Here we present the details: let $B_{R}\left(x_{k}\right)$ denote a Euclidean ball around $x_{k}$. According to Corollary 5.4, applied with $i=1$, we obtain for $x \in \tilde{B}_{R}\left(x_{k}\right)$ that the eigenvalues $\left(\lambda_{i}\left(x, t_{k}\right)\right)$ differ at most by $c \cdot R \cdot t_{k}^{-1 / 2}$ from the eigenvalues $\left(\lambda_{i}\left(x_{k}, t_{k}\right)\right)$,

$$
\sup _{i \in\{1, \ldots, n\}}\left|\lambda_{i}\left(x_{k}, t_{k}\right)-\lambda_{i}\left(x, t_{k}\right)\right| \leq \frac{c_{1} R}{\sqrt{t_{k}}} .
$$

We deduce that in a ball of radius at least $R$ around $x_{k}$ with $R:=\frac{1}{c_{1}} \zeta \sqrt{t_{k}}$, we have

$$
\max _{i \in\{1, \ldots, n\}}\left|\lambda_{i}\left(x, t_{k}\right)-1\right| \geq \zeta
$$

Note that this implies

$$
\max _{i \in\{1, \ldots, n\}}\left|\lambda_{i}^{m}(x, t)-1\right| \geq \zeta
$$


So at least one eigenvalue differs significantly from one in that ball. As our solution is $\tilde{\varepsilon}(n)$-close to $h$ with $0<\tilde{\varepsilon}(n) \leq 1$, we still have

$$
\frac{1}{2} \leq(1+\tilde{\varepsilon})^{-1} \leq \lambda_{i}, \lambda_{i}^{-1} \leq 1+\tilde{\varepsilon} \leq 2
$$

We obtain in $B_{R}\left(x_{k}\right) \times\left\{t_{k}\right\}$

$$
\varphi_{m}+\psi_{m}-2 n=\sum_{i=1}^{m} \frac{1}{\lambda_{i}^{m}}\left(\lambda_{i}^{m}-1\right)^{2} \geq \frac{1}{2^{m}} \zeta^{2} .
$$

We define $\delta:=\frac{1}{2} \frac{1}{2^{m}} \zeta^{2}$ and get in $B_{R}\left(x_{k}\right) \times\left\{t_{k}\right\}$

$$
\begin{array}{r}
\varphi_{m}+\psi_{m}-2 n \geq 2 \delta \\
\left(\varphi_{m}+\psi_{m}-2 n-\delta\right)_{+} \geq \delta
\end{array}
$$

This allows to estimate $I_{\delta}^{m, p}\left(t_{k}\right)$ from below as follows:

$$
\begin{aligned}
I_{\delta}^{m, p}\left(t_{k}\right) & \geq \frac{1}{p} \int_{B_{\frac{1}{c_{1}} \zeta \sqrt{t_{k}}}\left(x_{k}\right)}\left(\varphi_{m}+\psi_{m}-2 n-\delta\right)_{+}^{p} \\
& \geq \frac{1}{p} \cdot \delta^{p} \cdot c(n) \cdot\left(\frac{1}{c_{1}} \zeta \sqrt{t_{k}}\right)^{n} .
\end{aligned}
$$

We deduce that $I_{\delta}^{m, p}\left(t_{k}\right) \rightarrow \infty$ for $t_{k} \rightarrow \infty$. This contradicts Theorem 6.1 which implies that

$$
I_{\delta}^{m, p}\left(t_{k}\right) \leq I_{\delta}^{m, p}(0)
$$

as our initial decay assumption guarantees that for fixed $m \in \mathbb{N}_{+}$as in Theorem $6.1, p \geq 1$, and $\delta>0$, the integral $I_{\delta}^{m, p}(0)$ is finite.

Proof of Theorem 1.3. Lemma 7.1 implies that $g(t) \rightarrow h$ in $C^{0}$ as $t \rightarrow \infty$. The convergence of the derivatives ${ }^{h} \nabla^{m} g(t)$ for all $m \in \mathbb{N}$ is a consequence of Corollary 5.4.

If $I_{0}^{m, p}(0)$ is finite for some $m, p$ as in Section 6 , such a calculation implies even a decay rate in time of

$$
\sup _{x \in \mathbb{R}^{n}}\left|\lambda_{i}(x, t)-1\right|
$$

Lemma 7.2. Let $g \in \mathcal{M}_{\mathrm{loc}}^{\infty}\left(\mathbb{R}^{n},(0, \infty)\right)$ be a solution to $(1.1)$ with $g_{0}$ as in Theorem 1.3, such that $g(t)$ is $\tilde{\varepsilon}(n)$-close to $h$ for all $t$. Then the eigenvalues 
$\left(\lambda_{i}\right)_{1 \leq i \leq n}$ of $g(t)$ with respect to the flat background metric $h$ converge to 1 as $t \rightarrow \infty$ :

$\sup _{x \in \mathbb{R}^{n}} \sup _{i \in\{1, \ldots, n\}}\left|\lambda_{i}(x, t)-1\right| \leq c\left(n, m, p, c_{1}\right) \cdot\left(I_{0}^{m, p}(0)\right)^{1 /(2 p+n)} \cdot\left(\frac{1}{t}\right)^{n /(2(2 p+n))}$ if $I_{0}^{m, p}(0)<\infty$.

Proof. Fix $x_{0} \in \mathbb{R}^{n}, t_{0}>0$, and $i_{0} \in\{1, \ldots, n\}$. Define

$$
\zeta:=\frac{1}{2}\left|\lambda_{i_{0}}\left(x_{0}, t_{0}\right)-1\right| .
$$

We want to estimate $\zeta$ from above. As in the proof of Lemma 7.1, we obtain that

$$
\begin{aligned}
\zeta & \leq \max _{i \in\{1, \ldots, n\} x \in B_{\zeta \sqrt{t_{0}} / c_{1}}\left(x_{0}\right)}\left|\lambda_{i}\left(x, t_{0}\right)-1\right|, \\
\varphi_{m}+\psi_{m}-2 n & \geq \frac{1}{2^{m}} \zeta^{2} \\
I_{0}^{m, p}(0) & \geq I_{0}^{m, p}\left(t_{0}\right) \\
& \geq \frac{1}{p} \int_{x \in B_{\zeta \sqrt{t_{0} / c_{1}}}\left(x_{0}\right)}\left(\varphi_{m}+\psi_{m}-2 n\right)^{p} \\
& \geq \frac{1}{p}\left(\frac{1}{2^{m}} \zeta^{2}\right)^{p} \cdot c(n) \cdot\left(\frac{1}{c_{1}} \zeta \sqrt{t_{0}}\right)^{n}
\end{aligned}
$$

and thus

$$
(2 \zeta)^{2 p+n} \leq c\left(n, m, p, c_{1}\right) \cdot t_{0}^{-n / 2} \cdot I_{0}^{m, p}(0)
$$

Our claim follows.

\section{Improved $C^{1}$-estimates}

Based on an iteration of two steps, we can improve our a priori estimates. So far, we have obtained a priori estimates of the form

$$
|g-h|_{C^{0}} \leq \frac{c\left(n, m, p, c_{1}(n), I_{0}^{m, p}\right)}{t^{\frac{n}{2(2 p+n)}}} \equiv \frac{c_{0}}{t^{\frac{n}{2(2 p+n)}}}
$$


see Lemma 7.2, and

$$
\left|{ }^{h} \nabla^{i} g\right| \leq \frac{c(i, n)}{t^{i / 2}} \equiv \frac{c_{i}}{t^{i / 2}}
$$

for every $i \in \mathbb{N}$, see Lemma 5.4. The two steps to improve these are as follows.

Step 1: Interpolation inequalities of the form $\|D u\|_{L^{\infty}}^{2} \leq c \cdot\|u\|_{L^{\infty}}$. $\left\|D^{2} u\right\|_{L^{\infty}}$ and an iteration argument as in [11, Lemma C.2] can be applied to a metric $g$ satisfying (8.1) and

$$
|g-h|_{C^{0}} \leq \frac{a}{t^{\alpha}}
$$

for some constant $a$. We obtain for any $0<\beta<\alpha$ the estimate

$$
\left|{ }^{h} \nabla^{i} g\right| \leq \frac{c\left(\left(c_{k}\right)_{1 \leq k \leq k_{0}(i, \alpha, \beta)}, a, \alpha, \beta\right)}{t^{(i / 2)+\beta}},
$$

$c_{k}$ as in (8.1). Note that if $\beta$ gets closer to $\alpha$, the upper bound depends on more and more constants $c_{k}$ as in (8.1).

Step 2: Recall that for solutions of the $h$-flow satisfying

$$
\left|{ }^{h} \nabla g\right| \leq \frac{c}{t^{\gamma}}
$$

arguing as in Lemma 7.2 yields

$$
|g-h|_{C^{0}} \leq \frac{c}{t^{\gamma\left(\frac{n}{2 p+n}\right)}} .
$$

Iteration. We start with

$$
\left|{ }^{h} \nabla g\right| \leq \frac{c}{t^{\gamma_{0}}}
$$

According to Corollary 5.4 (with $i=1$ ), we may take $\gamma_{0}=\frac{1}{2}$. So Step 2 yields

$$
|g-h|_{C^{0}} \leq \frac{C_{1}}{t^{\alpha_{1}}}
$$

with $\alpha_{1}=\frac{n}{2(2 p+n)}$. By combining Steps 1 and 2, we get

$$
|g-h|_{C^{0}} \leq \frac{C_{2}(\delta)}{t^{\alpha_{2}}}
$$


with $\alpha_{2}=\left(\frac{1}{2}+(1-\delta) \alpha_{1}\right) \frac{n}{2 p+n}$ for any fixed $0<\delta<1$. We also get

$$
|g-h|_{C^{0}} \leq \frac{C_{k}(\delta)}{t^{\alpha_{k}}}
$$

with $\alpha_{k+1}=\left(\frac{1}{2}+(1-\delta) \alpha_{k}\right) \frac{n}{2 p+n}$ for all $k \in \mathbb{N}$.

It is easy to check that

$$
\frac{n}{2(2 p+n)}=\alpha_{1}<\alpha_{2}<\ldots<\frac{n}{2(2 p+\delta n)} .
$$

As $\alpha_{k}$ is increasing in $k$, we see that $\lim \alpha_{k}$ exists. Passing to the limit in the defining equation for $\alpha_{k}$ yields

$$
\lim _{k \rightarrow \infty} \alpha_{k}=\frac{n}{2(2 p+\delta n)}
$$

Now $\delta>0$ can be chosen arbitrarily small. So we obtain for any $0<\zeta \ll 1$

$$
|g-h|_{C^{0}} \leq \frac{c(\zeta)}{t^{n /(4 p)-\zeta}}
$$

We can finally apply step 1 once again in order to obtain a derivative bound.

Thus we have proved the following.

Lemma 8.1. Let $g$ be as in Lemma 7.2. Then we obtain for every $0<\zeta \ll 1$ the estimates

$$
|g-h|_{C^{0}} \leq \frac{c(\zeta)}{t^{n /(4 p)-\zeta}}
$$

and for every $i \in \mathbb{N}$

$$
\left|{ }^{h} \nabla^{i} g\right| \leq \frac{c(\zeta)}{t^{i / 2+n /(4 p)-\zeta}}
$$

\section{Construction of a solution to Ricci flow}

In this section we construct a solution to $\mathrm{RF}$ for smooth initial metrics $g_{0}$ on $\mathbb{R}^{n}$ which are $\varepsilon$-close to the standard metric $\delta$. It is well known that on a compact manifold, we can recover the RF from a solution to the $h$-flow using time-dependent diffeomorphisms $[7,14]$. We use the a priori estimates of the previous section and construct diffeomorphisms in our set up which allow us to construct a solution to the RF (similar to the compact case). 
Lemma 9.1. Let $g \in \mathcal{M}_{\mathrm{loc}}^{\infty}\left(\mathbb{R}^{n},[0, \infty)\right)$ be a solution to (1.1) which is $\tilde{\varepsilon}(n)$-close to $h$ (for all $t$ with $\tilde{\varepsilon}$ as in Theorem 1.2). Then there exists a smooth family of diffeomorphisms $(\varphi(\cdot, t))_{t \in[0, \infty)}, \varphi_{t} \equiv \varphi(\cdot, t): \mathbb{R}^{n} \rightarrow \mathbb{R}^{n}$, with $\varphi(x, 0)=x$, such that $\left(\varphi_{t}\right)^{*}(g(t))$ solves $(\mathrm{RF})$.

Proof. Let ${ }^{i} \varphi: B_{i} \times[0, \infty) \rightarrow B_{i} \subset \mathbb{R}^{n}$ be the solutions to the ordinary differential equations

$$
\begin{cases}\frac{\partial}{\partial t}\left({ }^{i} \varphi\right)^{\alpha}(x, t)=\eta_{i}(\varphi(x, t)) V^{\alpha}(\varphi(x, t), t), & (x, t) \in B_{i} \times[0, \infty), \\ { }^{i} \varphi(x, 0)=x, & x \in B_{i}\end{cases}
$$

where $V^{\alpha}(y, t):=g^{\beta \gamma}\left({ }^{g} \Gamma_{\beta \gamma}^{\alpha}-{ }^{h} \Gamma_{\beta \gamma}^{\alpha}\right)(y, t)$, and $\eta_{i}: \mathbb{R}^{n} \rightarrow \mathbb{R}$ is a smooth cutoff function $0 \leq \eta \leq 1$ with $\left.\eta\right|_{B_{i / 2}} \equiv 1$ and $\left.\eta\right|_{\mathbb{R}^{n}-B_{i}} \equiv 0$. Notice that ${ }^{i} V:=$ $\eta_{i} V$ is smooth and compactly supported in $B_{i}$. Hence the $\varphi_{i}(\cdot, t): B_{i} \rightarrow B_{i}$ are diffeomorphisms.

Using again Corollary 5.4 we see that for $x \in B_{i / 2}$ and small times

$$
\left|{ }^{h} \nabla g(x, t)\right| \leq \frac{c(n)}{\sqrt{t}}
$$

This implies that

$$
\left|\frac{\partial}{\partial t}\left({ }^{i} \varphi\right)^{\alpha}(x, t)\right|=\left|{ }^{i} V^{\alpha}\left({ }^{i} \varphi(x, t), t\right)\right| \leq \frac{c(n)}{\sqrt{t}}
$$

(here we have used that $g(t)$ is $\varepsilon$-close to $h$ ). In particular, this gives us

$$
\left|\left({ }^{i} \varphi\right)^{\alpha}(x, t)-x\right| \leq \int_{0}^{t}\left|\frac{\partial}{\partial \tau}\left({ }^{i} \varphi\right)^{\alpha}(x, \tau)\right| d \tau \leq c(n) \sqrt{t}
$$

This implies that $\left.{ }^{i} \varphi(\cdot, t)\right|_{B_{j}}$ is independent of $i$ for $i$ big enough, $j$ fixed and $t$ bounded above. In particular we obtain, taking a (diagonal subsequence) limit, a well-defined solution $\varphi: \mathbb{R}^{n} \times[0, \infty) \rightarrow \mathbb{R}^{n}$ to the equation

$$
\begin{cases}\frac{\partial}{\partial t}(\varphi)^{\alpha}(x, t)=V^{\alpha}(\varphi(x, t), t), & (x, t) \in \mathbb{R}^{n} \times[0, \infty) \\ \varphi(x, 0)=x, & x \in \mathbb{R}^{n}\end{cases}
$$

and thus $\varphi_{t}=\varphi(t, \cdot): \mathbb{R}^{n} \rightarrow \mathbb{R}^{n}$ is a diffeomorphism for all $t$. According to $[12]\left(\varphi_{t}\right)^{*}(g(t))$ solves the RF equation. 
In the situation that we have the improved decay estimates of Lemma 8.1 we show that the diffeomorphisms $\varphi_{t}$ converge to a diffeomorphism $\varphi_{\infty}: \mathbb{R}^{n} \rightarrow$ $\mathbb{R}^{n}$ as $t \rightarrow \infty$ for $n \geq 3$ as long as $p \geq 1$ is small enough.

Theorem 9.2. Let $g \in \mathcal{M}_{\mathrm{loc}}^{\infty}\left(\mathbb{R}^{n},[0, \infty)\right)$ be a solution to (1.1) and $m, p$ and $\tilde{\varepsilon}(m, n)$ all be as in Theorem 6.1. Assume that (1.5) holds. For $n \geq 3$, $\varphi_{t}$ converges in $C_{\mathrm{loc}}^{\infty}$ to a diffeomorphism $\varphi_{\infty}: \mathbb{R}^{n} \rightarrow \mathbb{R}^{n}$ as $t \rightarrow \infty$.

Proof. Step 1. Fix $p$ with $n / 2>p \geq 1$ and $\zeta>0$ such that $\alpha:=(1 / 2)+n /(4 p)$ $-\zeta>1$. In the following, our constants $c$ may depend on $n, p, \zeta, m$ and $I_{0}^{m, p}$ as defined in Theorem 6.1.

We wish to show that

$$
\left|\varphi_{t}(x)-x\right| \leq c \quad \forall t \in[0, \infty)
$$

Corollary 5.4 implies that

$$
\left|\frac{d}{d t} \varphi_{t}(x)\right| \leq \frac{c}{t^{1 / 2}}
$$

Hence $\left|\varphi_{t}(x)-x\right| \leq c$ for all $0 \leq t \leq 1$. So we may assume that $t>1$. The evolution equation (9.1) for $\varphi_{t}$ and the fact that $|\nabla g| \leq \frac{c}{t^{\alpha}}$ imply that

$$
\left|\frac{d}{d t} \varphi_{t}(x)\right| \leq \frac{c}{t^{\alpha}}
$$

As $\alpha>1$, we see that (upon integrating from 1 to $t>1$ ) $\left|\varphi_{1}(x)-\varphi_{t}(x)\right| \leq c$. By the triangle inequality we get

$$
\left|\varphi_{t}(x)-x\right| \leq\left|\varphi_{t}(x)-\varphi_{1}(x)\right|+\left|\varphi_{1}(x)-x\right| \leq c
$$

For later use, note that this implies for $F_{t}: \mathbb{R}^{n} \rightarrow \mathbb{R}^{n}$ defined by $F_{t}:=\varphi_{t}^{-1}$

$$
\left|F_{t}(x)-x\right| \leq c
$$

Step 2. Using the estimate $\left|\frac{d}{d t} \varphi_{t}(x)\right| \leq \frac{c}{t^{\alpha}}$ from Step 1, we see that for fixed $x \in \mathbb{R}^{n}, \varphi_{t}(x) \rightarrow y$ as $t \rightarrow \infty$ for some $y \in \mathbb{R}^{n}$. We define 
$\varphi_{\infty}(x)=\lim _{t \rightarrow \infty} \varphi_{t}(x)$. All the derivatives of $\varphi_{t}$ (for $\left.t>1\right)$ satisfy

$\frac{\partial}{\partial t} \log \left(1+\left|{ }^{h} \nabla^{m} \varphi_{t}(x)\right|^{2}\right) \leq \frac{c\left(\left.\varphi_{1}\right|_{B_{r}(y)}, n, m, p, \zeta,\left(c_{k}\right), I_{0}^{m, p}\right)}{t^{\alpha}} \quad \forall x \in B_{r}(y)$

for some $\alpha>1$ and

$$
\sup _{x \in B_{r}(y)}\left|{ }^{h} \nabla^{m} \varphi_{t}(x)\right|^{2} \leq c\left(\left.\varphi_{1}\right|_{B_{r}(y)}, n, m, p, \zeta,\left(c_{k}\right), I_{0}^{m, p}\right)
$$

This may be seen as follows (for the rest of this argument, we set $\nabla:={ }^{h} \nabla$ ).

Differentiating gives for $V$ as in (1.1)

$$
\left|\nabla^{m} V(x, t)\right| \leq c_{m} \sum_{k=1}^{m+1} \sum_{\substack{i_{1}+\cdots+i_{k}=m+1 \\ 1 \leq i_{1}, \ldots, i_{k} \leq m+1}}\left|\nabla^{i_{1}} g\right| \cdots\left|\nabla^{i_{k}} g\right|,
$$

$\left|\nabla^{m}(V(\varphi(x, t), t))\right| \leq c_{m} \sum_{i=1}^{m} \sum_{\substack{j_{1}+\ldots+j_{i}=m \\ 1 \leq j_{1}, \ldots, j_{i} \leq m}}\left|\left(\nabla^{i} V\right)(\varphi(x, t), t)\right| \cdot\left|\nabla^{j_{1}} \varphi\right| \cdots\left|\nabla^{j_{i}} \varphi\right|$.

Then

$$
\begin{aligned}
\frac{\partial}{\partial t} \log \left(1+\left|\nabla^{m} \varphi_{t}(x)\right|^{2}\right) & =\frac{\frac{\partial}{\partial t}\left|\nabla^{m} \varphi_{t}(x)\right|^{2}}{\left(1+\left|\nabla^{m} \varphi_{t}(x)\right|^{2}\right)} \\
& \leq c \frac{\left|\nabla^{m} \varphi_{t}(x)\right|\left|\nabla^{m} \frac{\partial}{\partial t} \varphi_{t}(x)\right|}{\left(1+\left|\nabla^{m} \varphi_{t}(x)\right|^{2}\right)} \\
& =c \frac{\left|\nabla^{m} \varphi_{t}(x)\right|\left|\nabla^{m}\left(V\left(\varphi_{t}, t\right)\right)(x)\right|}{\left(1+\left|\nabla^{m} \varphi_{t}(x)\right|^{2}\right)}
\end{aligned}
$$

Substituting (9.2) and (9.3) into the above, using the estimates for $\left|\nabla^{k} g\right|$ and arguing inductively (for $m$ ) leads to the estimate.

Hence $\varphi_{\infty}: \mathbb{R}^{n} \rightarrow \mathbb{R}^{n}$ is $C^{\infty}$ and $\varphi_{t} \rightarrow \varphi_{\infty}$ converges in $C_{\mathrm{loc}}^{\infty}$ as $t \rightarrow \infty$.

Step 3. We wish to show that $\varphi_{\infty}$ is a diffeomorphism. Let $F_{t}$ be the inverse of $\varphi_{t}$. Letting $l(t):=\varphi_{t}^{*} g(t)$, we know that $l$ solves $\mathrm{RF}$ on $\mathbb{R}^{n}$. But 
then we get in view of $(8.2)$

$$
\left|\frac{\partial}{\partial t} l\right|(x, t)=2 \cdot{ }^{l}|\operatorname{Ric}(l)|(x, t)=2 \cdot{ }^{g}|\operatorname{Ric}(g)|\left(\varphi_{t}(x), t\right) \leq \frac{c}{t^{3 / 2}} \quad \text { for } t \geq 1,
$$

which is integrable (from 1 to $\infty$ ). This implies that $l(t)$ converges locally uniformly to a well-defined continuous Riemannian metric $l_{\infty}$ as $t \rightarrow \infty$ in view of [5, Lemma 14.2]. According to [5], there must exist a $c=c(y)$ with $\frac{1}{c} h(x) \leq l(x, t) \leq c h(x)$ for all $t \in[0, \infty)$ for all $x \in B_{1}(y)$, for all $y \in \mathbb{R}^{n}$, as Theorem 4.4 implies this uniform equivalence for $t \in[0,1]$.

Now using the definition of $l$ we get

$$
\begin{aligned}
\frac{1}{c} \delta_{\alpha \beta} & \leq l_{\alpha \beta}(x, t)=\frac{\partial \varphi^{s}}{\partial y^{\alpha}}(x, t) \frac{\partial \varphi^{k}}{\partial y^{\beta}}(x, t) g_{s k}\left(\varphi_{t}(x), t\right) \\
& \leq(1+\tilde{\varepsilon}) \frac{\partial \varphi^{s}}{\partial y^{\alpha}} \frac{\partial \varphi^{k}}{\partial y^{\beta}}(x, t) \delta_{s k} \\
& =(1+\tilde{\varepsilon})(D \varphi)(D \varphi)^{T}(x, t)
\end{aligned}
$$

In particular, we see that $\operatorname{det}\left(D \varphi_{t}\right)^{2}(x) \geq 1 /(c(1+\tilde{\varepsilon}))>0$, where $D f$ is the Jacobian of $f$. Taking the limit as $t \rightarrow \infty$ we get $\operatorname{det}\left(D \varphi_{\infty}\right)^{2}(y) \geq 1 /(c(1+$ $\tilde{\varepsilon}))>0$. As $\operatorname{det}\left(D \varphi_{0}\right)=1$, we see that $\operatorname{det}\left(D \varphi_{\infty}\right)(y) \geq 1 / \sqrt{(1+\tilde{\varepsilon}) c(y)}>0$ for all $y \in \mathbb{R}^{n}$. Hence $\varphi_{\infty}$ is an immersion. In view of the derivative estimates for $\left|\nabla^{m} \varphi_{t}\right|$, the function $\varphi_{\infty}: \mathbb{R}^{n} \rightarrow \mathbb{R}^{n}$ is a smooth diffeomorphism if it is bijective. Recall that $F_{t} \equiv F(\cdot, t)=\left(\varphi_{t}\right)^{-1}$ for $t<\infty$. The estimates for $\varphi_{t}$ above ensure that there exists a function $F_{\infty}: \mathbb{R}^{n} \rightarrow \mathbb{R}^{n}$ such that $F_{t} \rightarrow F_{\infty}$ in $C_{\mathrm{loc}}^{\infty}$ as $t \rightarrow \infty$.

As $\varphi_{t}$ and $F_{t}$ are smooth diffeomorphisms with estimated derivatives, $\varphi_{t} \rightarrow \varphi_{\infty}$ and $F_{t} \rightarrow F_{\infty}$, we obtain

$$
\left(F_{\infty} \circ \varphi_{\infty}\right)(x)=\lim _{t \rightarrow \infty}\left(F_{t} \circ \varphi_{t}\right)(x)=x \quad \text { for all } x \in \mathbb{R}^{n}
$$

Thus $\varphi_{\infty}$ is injective. Similarly, we see that $\varphi_{\infty}$ is surjective. Hence $\varphi_{\infty}$ is a diffeomorphism.

Proof of Theorem 1.4. In Lemma 9.1 we have constructed diffeomorphisms $\varphi_{t}$ so that $\left(\varphi_{t}^{*} g(t)\right)_{t \in[0, \infty)}$ solves the RF equation if $(g(t))_{t \in[0, \infty)}$ solves $(1.1)$. Define $\tilde{g}(t):=\varphi_{t}^{*} g(t)$. According to Theorem 1.3, we get $g(t) \rightarrow h$. Theorem 9.2 implies that the diffeomorphisms $\varphi_{t}$ converge to a diffeomorphism $\varphi_{\infty}$ for $t \rightarrow \infty$. Thus $\tilde{g}(t) \rightarrow \varphi_{\infty}^{*} h$ in $C^{\infty}$ as $t \rightarrow \infty$. 
We can also show that the diffeomorphisms $\varphi_{t}$ are close to the identity near infinity, uniformly in $t$.

Lemma 9.3. Let $g \in \mathcal{M}_{\mathrm{loc}}^{\infty}\left(\mathbb{R}^{n},[0, \infty)\right)$ be a solution to $(1.1)$ as in Theorem 9.2 and $\left(\varphi_{t}\right)_{t \in[0, \infty]}$ be the diffeomorphisms of $\mathbb{R}^{n}$ constructed before. Then for every $\eta>0$ there exists an $R>0$ such that

$$
\sup _{\mathbb{R}^{n} \backslash B_{R}(0)}\left|\varphi_{t}(x)-x\right| \leq \eta
$$

for all $t \in[0, \infty]$.

Proof. We first want to show that for any given $\eta^{\prime}>0$ there exists an $R_{1}>0$ such that $g(t)$ is $\eta^{\prime}$-close to $h=\delta$ on $\mathbb{R}^{n} \backslash B_{R_{1}}(0)$ for all $t$.

By Lemma 7.2 we can choose a $T>0$ such that $g(t)$ is $\eta^{\prime}$-close to $h$ on all of $\mathbb{R}^{n}$ for $t \geq T$. Now choose $R_{0}>0$ such that $g(0)$ is $\eta^{\prime} / 2$-close to $h$ on $\mathbb{R}^{n} \backslash B_{R_{0}}(0)$. Applying the interior closeness estimates, Lemma 5.2, we see that we can find $R_{1}>R_{0}$ such that $g(t)$ is $\eta^{\prime}$-close to $h$ on $\left(\mathbb{R}^{n} \backslash B_{R_{1}}(0)\right) \times$ $[0, T]$. Note that this implies

$$
\sup _{\mathbb{R}^{n} \backslash B_{R_{1}}(0)}|g(t)-\delta| \leq \eta^{\prime}
$$

for all $t$. By Lemma 8.1 we have that

$$
\left|{ }^{h} \nabla g\right| \leq \frac{c}{t^{1 / 2+n /(4 p)-\zeta}}
$$

for $t>0$. Now fix $t_{1}>0$ such that

$$
\int_{t_{1}}^{\infty} \frac{c}{t^{1 / 2+n /(4 p)-\zeta}} d t \leq \frac{\eta}{2}
$$

By interpolation we deduce from Lemma 5.4 that

$$
\left|{ }^{h} \nabla g\right| \leq \frac{\delta\left(\eta^{\prime}\right)}{\sqrt{t}} \quad \text { on } \mathbb{R}^{n} \backslash B_{R_{1}}(0)
$$

for $t>0$, and in particular for $0<t \leq t_{1}$, where $\delta(\eta) \rightarrow 0$ as $\eta \rightarrow 0$. Arguing as in Step 1 of the proof of Theorem 9.2 we obtain the claimed estimate for some $R>R_{1}$. 


\section{Convergence based on integral bounds}

Proof of Theorem 1.6. In view of Corollary 5.4 and interpolation inequalities of the form $\|\nabla v\|_{C^{0}} \leq c \cdot\|v\|_{C^{0}} \cdot\|v\|_{C^{2}}$, it suffices to prove that $g(t) \rightarrow h$ in $C^{0}$ as $t \rightarrow \infty$.

Assume the conclusions were false. Then we could find $\delta>0$ and points

$$
\left(x_{k}, t_{k}, l_{k}\right) \in \mathbb{R}^{n} \times(0, \infty) \times\{1, \ldots, n\},
$$

$k \in \mathbb{N}$, such that $t_{k} \rightarrow \infty$ as $k \rightarrow \infty$ and

$$
\left|\lambda_{l_{k}}\left(x_{k}, t_{k}\right)-1\right| \geq 4 \delta
$$

By the construction of the solution $g(t)$ in Section 4 from solutions ${ }^{i} g$, we find sequences $\left(i_{j}^{k}\right)_{j \in \mathbb{N}}$ with $i_{j}^{k} \rightarrow \infty$ as $j \rightarrow \infty$, such that for the eigenvalues $\left({ }^{i} \lambda_{l}\right)_{1 \leq l \leq n}$ of ${ }^{i} g$, we have

$$
\left|i_{j}^{k} \lambda_{l_{k}}\left(x_{k}, t_{k}\right)-1\right| \geq 3 \delta \text {. }
$$

Fix $R>0$. We want to establish a similar bound on the spatial ball $B_{R}\left(x_{k}\right) \times$ $\left\{t_{k}\right\}$. This is done as follows. We consider only $k \geq k_{0}$ so that the $t_{k}$ is sufficiently large and hence the right-hand side in the estimate of the first spatial derivative $(i=1)$ in Corollary 5.4 is bounded above by $\delta / R$. Then we choose $R_{k} \geq R$ such that $\gamma\left(2 R_{k}\right)^{2} \geq t_{k}$, where $\gamma$ is as in the corollary. Now we fix $j=j(k)$ so large that $\nu_{k} \equiv i_{j(k)}^{k} \geq 2 R_{k}+\left|x_{k}\right|+1$. This ensures that the metric ${ }^{\nu_{k}} g$ is defined on the cylinder $B_{2 R_{k}}\left(x_{k}\right) \times\left(0, \gamma\left(2 R_{k}\right)^{2}\right)$. Hence we can apply Corollary 5.4 for that cylinder and get the gradient estimate $\left|{ }^{h} \nabla^{\nu_{k}} g\left(\cdot, t_{k}\right)\right| \leq \delta / R$ in $B_{R}\left(x_{k}\right)$. In view of (10.1), we obtain

$$
\left.\inf _{x \in B_{R}\left(x_{k}\right)} \sup _{1 \leq l \leq n}\right|^{\nu_{k}} \lambda_{l}\left(x, t_{k}\right)-1 \mid \geq 2 \delta
$$

Define

$$
{ }^{i} I_{\delta}^{m, p}(t):=\frac{1}{p} \int_{B_{i}(0)}\left({ }^{i} \varphi_{m}+{ }^{i} \psi_{m}-2 n-\delta\right)_{+}^{p} d x
$$

where

$$
{ }^{i} \varphi_{m}(x, t):=\sum_{l=1}^{n} \frac{1}{i \lambda_{l}^{m}(x, t)} \quad \text { and } \quad{ }^{i} \psi_{m}(x, t):=\sum_{l=1}^{n}{ }^{i} \lambda_{l}^{m}(x, t)
$$


By construction of the initial metrics ${ }^{i} g_{0}$, we have for the eigenvalues $\left(\lambda_{l}(x)\right)_{1 \leq l \leq n}$ of the metric $g_{0}$ with respect to $h$ which we label such that $\lambda_{1}(x) \leq \ldots \leq \lambda_{n}(x)$ and similarly for the eigenvalues ${ }^{i} \lambda_{1}(x) \leq \ldots \leq{ }^{i} \lambda_{n}(x)$ of ${ }^{i} g_{0}$ for $|x| \leq i$ the inequalities

$$
\begin{cases}\lambda_{l}(x) \leq{ }^{i} \lambda_{l}(x) \leq 1 & \text { if } \lambda_{l}(x) \leq 1 \\ 1 \leq{ }^{i} \lambda_{l}(x) \leq \lambda_{l}(x) & \text { if } 1 \leq \lambda_{l}(x)\end{cases}
$$

which implies in particular the estimate $\left({ }^{i} \lambda_{l}^{m}-1\right)^{2} \leq\left(\lambda_{l}^{m}-1\right)^{2}$ for all $i, l, m \in \mathbb{N}$. We deduce that

$$
\begin{aligned}
{ }^{i} \varphi_{m}+{ }^{i} \psi_{m}-2 n & =\sum_{l=1}^{n} \frac{1}{{ }^{i} \lambda_{l}^{m}}\left({ }^{i} \lambda_{l}^{m}-1\right)^{2} \\
& \leq \sum_{l=1}^{n} \frac{\lambda_{l}^{m}}{\lambda_{l}^{m}}(1+\varepsilon(n))^{m}\left(\lambda_{l}^{m}-1\right)^{2} \\
& \leq(1+\varepsilon(n))^{2 m} \sum_{l=1}^{n} \frac{1}{\lambda_{l}^{m}}\left(\lambda_{l}^{m}-1\right)^{2} \\
& =(1+\varepsilon(n))^{2 m}\left(\varphi_{m}+\psi_{m}-2 n\right) .
\end{aligned}
$$

Thus

$$
\begin{aligned}
{ }^{i} I_{\delta}^{m, p}(0) & =\left.\frac{1}{p} \int_{B_{i}(0)}\left({ }^{i} \varphi_{m}+{ }^{i} \psi_{m}-2 n-\delta\right)_{+}^{p} d x\right|_{t=0} \\
& \leq\left.(1+\varepsilon(n))^{2 m} \frac{1}{p} \int_{B_{i}(0)}\left(\varphi_{m}+\psi_{m}-2 n-\delta\right)_{+}^{p} d x\right|_{t=0} \\
& \leq\left.(1+\varepsilon(n))^{2 m} \frac{1}{p} \int_{\mathbb{R}^{n}}\left(\varphi_{m}+\psi_{m}-2 n-\delta\right)_{+}^{p} d x\right|_{t=0} \\
& =(1+\varepsilon(n))^{2 m} I_{\delta}^{m, p}(0)<\infty
\end{aligned}
$$

is uniformly bounded in $i$.

The proof of Theorem 6.1 extends directly to our situation and ensures that

$$
{ }^{i} I_{\delta}^{m, p}(t) \leq{ }^{i} I_{\delta}^{m, p}(0) \quad \text { for } t \geq 0 .
$$

Here, $\left({ }^{i} \varphi_{m}+{ }^{i} \psi_{m}-2 n-\delta\right)_{+}$is compactly supported in $B_{i}(0)$ due to the boundary values imposed on ${ }^{i} g$ in Theorem 4.3. Therefore, we do not have to use the local closeness estimates of Lemma 5.2 in order to justify the integration by parts employed in the proof of Theorem 6.1 . 
Using (10.2) and (10.3), we estimate for $\varepsilon$ and $m$ such that $(1+\varepsilon(n))^{m} \leq$ 2 as follows:

$$
\begin{aligned}
(1+\varepsilon(n))^{2 m} I_{\delta}^{m, p}(0) & \geq{ }^{\nu_{k}} I_{\delta}^{m, p}(0) \\
& \geq{ }^{\nu_{k}} I_{\delta}^{m, p}\left(t_{k}\right) \\
& =\frac{1}{p} \int_{B_{\nu_{k}}(0)}\left({ }^{\nu_{k}} \varphi\left(x, t_{k}\right)+{ }^{\nu_{k}} \psi\left(x, t_{k}\right)-2 n-\delta\right)_{+}^{p} d x \\
& \geq \frac{1}{p} \int_{x \in B_{R}\left(x_{k}\right)}\left(\sum_{l=1}^{n} \frac{1}{\nu_{k} \lambda_{l}^{m}\left(x, t_{k}\right)}\left({ }^{\nu_{k}} \lambda_{l}^{m}\left(x, t_{k}\right)-1\right)^{2}-\delta\right)_{+}^{p} d x \\
& \geq \frac{1}{p} \int_{B_{R}\left(x_{k}\right)}\left(\frac{1}{(1+\varepsilon(n))^{m}} 4 \delta-\delta\right)^{p} \\
& \geq \frac{1}{p} \int_{B_{R}\left(x_{k}\right)} \delta^{p} \\
& =\frac{\delta^{p}}{p} c(n) R^{n} .
\end{aligned}
$$

As $R \geq 0$ can be chosen arbitrarily large, we arrive at a contradiction. This finishes the proof of Theorem 1.6.

\section{Appendix: Conformal Ricci flow in two dimensions}

Studying RF on $\mathbb{R}^{2}$ for conformally flat metrics $g(x, t)=e^{u(x, t)} \delta$ is equivalent $[6,16]$ to considering the evolution equation

$$
\frac{\partial}{\partial t} u=\Delta_{g} u=e^{-u} \Delta_{\delta} u \quad \text { on } \mathbb{R}^{2} \times(0, \infty)
$$

We are especially interested in initial conditions $u_{0}: \mathbb{R}^{2} \rightarrow \mathbb{R}$ fulfilling

$$
\sup _{\mathbb{R}^{2} \backslash B_{r}(0)}\left|u_{0}\right| \rightarrow 0 \quad \text { as } r \rightarrow \infty
$$

We wish to point out that we do not assume in the following that $u_{0}$ fulfills an $\varepsilon_{0}$-closeness condition for a small constant $\varepsilon_{0}>0$, corresponding to $\sup \left|u_{0}\right|$ being small. It suffices to have sup $\left|u_{0}\right|$ bounded. Note also that in two dimensions, we do not have to study Ricci harmonic map heat flow first before we can obtain results for RF. 
In this situation, we get the following existence result.

Theorem A.1. Let $u_{0} \in C^{0}\left(\mathbb{R}^{2}\right)$ satisfy $\left\|u_{0}\right\|_{L^{\infty}}<\infty$. Then there exists $u \in C^{\infty}\left(\mathbb{R}^{2} \times(0, \infty)\right)$ solving $(\mathrm{A} .1)$ such that $u(\cdot, t) \rightarrow u_{0}$ in $C_{\mathrm{loc}}^{0}\left(\mathbb{R}^{2}\right)$ as $t \searrow 0$. As $t \rightarrow \infty, u(\cdot, t)$ converges subsequentially in $C_{\mathrm{loc}}^{\infty}$ to a complete flat metric.

Idea of Proof. Mollifying $u_{0}$, we get a sequence $u_{0}^{i} \in C^{\infty}\left(\mathbb{R}^{2}\right), i \in \mathbb{N}$, such that $u_{0}^{i} \rightarrow u_{0}$ in $C_{\mathrm{loc}}^{0}$ as $i \rightarrow \infty,\left\|u_{0}^{i}\right\|_{L^{\infty}} \leq\left\|u_{0}\right\|_{L^{\infty}}$, and $\left\|D^{2} u_{0}^{i}\right\|_{L^{\infty}}+$ $\left\|D u_{0}^{i}\right\|_{L^{\infty}} \leq c_{i}$. According to [16], there exist smooth solutions $u^{i}$ for $t \geq 0$ solving (A.1) with $u^{i}(\cdot, 0)=u_{0}^{i}$ such that $\left|u^{i}(x, t)\right| \leq \sup \left|u_{0}\right|$. The techniques of Krylov-Safonov, Schauder, and scaling imply $\left|D^{k} u^{i}\right| \leq \frac{c_{k}}{t^{k / 2}}$. Thus we find a smooth solution $u$ to (A.1). Considering $\psi=u^{i}(x, t)-u^{i}\left(x_{0}\right)$, we can argue as in Lemma 5.2 (using $\psi^{2}$ instead of $\varphi_{m}+\psi_{m}-2 n$ ) to see that for any $\delta>0$ we find $i_{0}=i_{0}\left(\delta, u_{0}\right)$ and $\zeta>0$ such that $\left|u^{i}(x, t)-u^{i}\left(x_{0}\right)\right| \leq \delta$ for $\left|x-x_{0}\right|^{2}+t \leq \zeta$ and $i \geq i_{0}$. Thus $u(\cdot, t) \rightarrow u_{0}$ in $C_{\mathrm{loc}}^{0}$ as $t \searrow 0$. Note that in the case of (A.2), this convergence is uniform in space. The remaining details are similar to the higher dimensional case.

Theorem A.2. Let $u_{0}$ be as in Theorem A.1 and assume that (A.2) is fulfilled. Let $u$ be the solution to (A.1) obtained there. Then $u(\cdot, t) \rightarrow 0$ in $C^{\infty}$ as $t \rightarrow \infty$.

Idea of Proof. The proof is similar to the proof of Theorem 1.3. Choose $p \geq$ $\sup \left|u_{0}\right|+1$. According to the construction of $u$ in the proof of Theorem A.1 and the maximum principle, we have $p \geq \sup _{x}\left|u_{0}(x)\right|+1 \geq \sup _{x, t}$ $|u(x, t)|+1$. Instead of Theorem 6.1 , however, we use the following estimate (and a similar argument for the negative part of $u$ )

$$
\begin{aligned}
\frac{d}{d t} \int_{\mathbb{R}^{2}} \frac{1}{p}(u-\delta)_{+}^{p} d x & =\int_{\{u>\delta\}}(u-\delta)^{p-1} e^{-u} \Delta_{\delta} u d x \\
& =\int_{\{u>\delta\}}(u-\delta)^{p-2}((u-\delta)-p+1) e^{-u}|\nabla u|^{2} d x \leq 0 .
\end{aligned}
$$

\section{Acknowledgments}

The authors want to thank Gerhard Huisken for fruitful discussions. The third author acknowledges support from SFB 647/B3 during his visits in Berlin. This paper is part of the research project "Stability of non-compact 
manifolds under curvature flows" of the first two authors within the priority program "Global Differential Geometry" SPP 1154 of the German research foundation DFG.

\section{References}

[1] A. Chau and O.C. Schnürer, Stability of gradient Kähler-Ricci solitons, Comm. Anal. Geom. 13(4) (2005), 769-800.

[2] B.-L. Chen and X.-P. Zhu, Uniqueness of the Ricci flow on complete noncompact manifolds, J. Differential Geom. 74(1) (2006), 119-154.

[3] J. Clutterbuck, O.C. Schnürer and F. Schulze, Stability of translating solutions to mean curvature flow, Calc. Var. Partial Differential Equations 29(3) (2007), 281-293.

[4] C. Guenther, J. Isenberg and D. Knopf, Stability of the Ricci flow at Ricci-flat metrics, Comm. Anal. Geom. 10(4) (2002), 741-777.

[5] R.S. Hamilton, Three-manifolds with positive Ricci curvature, J. Differential Geom. 17(2) (1982), 255-306.

[6] R.S. Hamilton, The Ricci flow on surfaces, Mathematics and general relativity (Santa Cruz, CA, 1986), Contemporary Mathematics, 71, Am. Math. Soc., Providence, RI, 1988, 237-262.

[7] R.S. Hamilton, The formation of singularities in the Ricci flow, Surveys in differential geometry, Vol. II (Cambridge, MA, 1993), Int. Press, Cambridge, MA, 1995, 7-136.

[8] S.-Y. Hsu, Uniqueness of solutions of Ricci flow on complete noncompact manifolds, arXiv:0704.3468v1 [math.DG].

[9] O.A. Ladyženskaja, V.A. Solonnikov and N.N. Ural'ceva, Linear and quasilinear equations of parabolic type, Translated from the Russian by S. Smith. Transl. Math. Monogr. 23, Am. Math. Soc., Providence, RI, 1967.

[10] T.A. Oliynyk and E. Woolgar, Rotationally symmetric Ricci flow on asymptotically flat manifolds, Comm. Anal. Geom. 15(3) (2007), 535-568.

[11] O.C. Schnürer and K. Smoczyk, Neumann and second boundary value problems for Hessian and Gauß curvature flows, Ann. Inst. H. Poincaré. Anal. Non Linéaire 20(6) (2003), 1043-1073. 
[12] W.-X. Shi, Deforming the metric on complete Riemannian manifolds, J. Differen. Geom. 30(1) (1989), 223-301.

[13] W.-X. Shi, Ricci deformation of the metric on complete noncompact Riemannian manifolds, J. Differential Geom. 30(2) (1989), 303-394.

[14] M. Simon, Deformation of $C^{0}$ Riemannian metrics in the direction of their Ricci curvature, Comm. Anal. Geom. 10(5) (2002), 1033-1074.

[15] N. Šešum, Linear and dynamical stability of Ricci flat metrics, Duke Math. J. 133(1) (2006), 1-26.

[16] L.-F. Wu, The Ricci flow on complete $\mathbf{R}^{2}$, Comm. Anal. Geom. 1(3-4) (1993), 439-472.

Freie Universität Berlin

Arnimallee 3

14195 BERLIN

GERMANY

E-mail address: oliver.schnuerer@math.fu-berlin.de

E-mail address: felix.schulze@math.fu-berlin.de

UNIVERSITÄT FREIBURG

ECKERSTRASSE 1

79104 FrEIBURG I. BR.

GERMANY

E-mail address: msimon@gmx.de

ReCEIVED June 07, 2007 